\title{
Two-phase entropy solutions of forward-backward parabolic problems with unstable phase
}

\author{
ANDREA TERRACINA \\ Dipartimento di Matematica "G. Castelnuovo”, Università di Roma "La Sapienza”, \\ Piazzale A. Moro, 2 - 00185 Roma, Italy \\ E-mail: terracin@mat.uniromal.it
}

[Received 9 May 2014 and in revised form 19 May 2015]

\begin{abstract}
In this paper we study a two-phase problem for a forward-backward parabolic equation with diffusion function of cubic type. Existence and uniqueness for these kind of problems were obtained in literature in the case in which the phases are both stable. Here we consider the situation in which the unstable phase is taken in account, obtaining not trivial solutions of the problem. It is interesting to note that such solutions are given by solving generalized Abel's equations.
\end{abstract}

2010 Mathematics Subject Classification: Primary 35K20; Secondary 35A01D2.

Keywords: Two-phase solutions, phase transition, forward-backward equations, ill-posed problems.

\section{Introduction}

In this paper we study the following forward-backward parabolic problem:

$$
\begin{cases}u_{t}=\phi(u)_{x x} & \text { in } Q_{T}:=\mathbb{R} \times(0, T) \\ u(x, 0)=u_{0}(x) & \text { in } \mathbb{R} \times\{0\}\end{cases}
$$

where $\phi$ is a nonmonotone function. This problem arises in different mathematical models in phase transition [5], population dynamics [31], [32], oceanography [1], image processing [33]. Obviously problem (1) is ill-posed whenever $u$ takes values in the interval in which $\phi$ decreases.

In this paper we focus to the model of phase transition where the response function $\phi$ is of "cubic type". More precisely, we assume that $\phi \in \operatorname{Li} p_{l o c}(\mathbb{R})$ and

$$
\lim _{u \rightarrow \pm \infty} \phi(u)= \pm \infty
$$

We suppose that $\phi$ has a local minimum $A$ and a local maximum $B$ such that $A<B$. Let us denote with $c$, respectively $b$, the point in which the local minimum $A$, respectively maximum $B$, is achieved. There are three regions $(-\infty, b),(c,+\infty)$ and $(b, c)$; the first two in which $\phi$ increases and the last one in which $\phi$ decreases.

In the phase transition models, the function $u$ gives the phase field, then the increasing intervals correspond to the stable phases and the interval $(b, c)$ to the unstable, or metastable one.

In this framework it is included also the piecewise linear case in which $\phi$ is given by

$$
\phi(u)= \begin{cases}\phi_{1}(u) & \text { for } u \leqslant b \\ \phi_{0}(u) & \text { for } b<u<c \\ \phi_{2}(u) & \text { for } u \geqslant c,\end{cases}
$$


where

$$
\phi_{i}(u):=\gamma_{i} u+\delta_{i}, i=0,1,2 .
$$

Here $-\infty<b<c<\infty, \gamma_{i}>0, i=1,2, \delta_{i} \in \mathbb{R}, A:=\phi_{2}(c)<\phi_{1}(b)=$ : B. In particular, $\phi_{0}(u):=\frac{A(u-b)-B(u-c)}{c-b}$.

In [20] (see also [22]), it was proved that uniqueness does not hold for problem (1) also in the class of solutions that take values only in the two stable phases.

Therefore, it is necessary to impose some stricter conditions in order to give a good formulation of the problem (1). The idea is to introduce a proper regularization that comes from the physical phenomena of the original model. A classical approximation term is that introduced by the CahnHilliard model that describes the cost of the inhomogeneities in phase transition. On the other hand, the mathematical description of the physical phenomena is much more complicated to that given by the Cahn-Hilliard equation and there are some other terms to take in account (see, e.g., [4, 5, 9, $19,45])$. As a matter of fact, it is possible to choose different types of regularization in which only some phenomena are highlighted (see e.g. [1-3, 6, 15, 17, 26, 30, 34, 35, 42]).

In this paper we refer to the following pseudoparabolic regularization

$$
\begin{cases}u_{t}=v_{x x} & \text { in } Q_{T}=\mathbb{R} \times(0, T) \\ u(x, 0)=u_{0}(x) & \text { in } \mathbb{R} \times\{0\},\end{cases}
$$

where $v=\phi(u)+\epsilon u_{t}, \epsilon>0$.

The third order term $\epsilon u_{t x x}$ is a viscosity term related to non-equilibrium effects (see, e.g., [4, $10,19])$.

In [30] the authors considered the following Neumann boundary problem

$$
\begin{cases}u_{t}=\Delta\left(\phi(u)+\epsilon u_{t}\right) & \text { in } Q_{T}=\Omega \times(0, T) \\ \frac{\partial}{d v}\left(\phi(u)+\epsilon u_{t}\right)=0 & \text { in } Q_{T}=\partial \Omega \times(0, T) \\ u(x, 0)=u_{0}(x) & \text { in } \Omega \times\{0\},\end{cases}
$$

where $\Omega \subset \mathbb{R}^{n}$ is a bounded "regular" domain. They obtained global existence and uniqueness for a large class of initial data. Moreover, they proved that the solutions satisfy some viscous entropy inequalities. These inequalities are crucial to obtain a priori estimates that do not depend on the parameter $\epsilon$.

The singular limit of problems (4) was analyzed by Plotnikov (see [34-36]). Using that approach, the author obtained an entropy formulation for the solutions of the original forwardbackward problem. The idea was to assume that the entropy solutions of the original forwardbackward problem are that obtained as limit of solutions of the problems (4) when $\epsilon$ goes to $0^{+}$(see also [28, 44]). We do not enter in the details of the formulation given by Plotnikov (see Section 2). However, it is important to mention that the original forward-backward equation is not more satisfied. Just to give an idea, we can extract subsequences $\left\{u_{\epsilon_{n}}\right\}$ of solutions of the viscous problems (4) converging in the weak* topology of $L^{\infty}$ to a function $u$ and a corresponding sequence $\left\{v_{\epsilon_{n}}\right\}$, defined by $v_{\epsilon_{n}}=\phi\left(u_{\epsilon_{n}}\right)+\epsilon_{n}\left(u_{\epsilon_{n}}\right)_{t}$, converging to $v$, such that, in general, $v \neq \phi(u)$. Then, we have $u_{t}=v_{x x}$ in a weak way. Therefore, the original forward-backward equation does not hold generally. Plotnikov gave in [35] a characterization of the Young measure associated to the converging sequence $\left\{u_{\epsilon_{n}}\right\}$. More precisely, he proved that the Young measure is given by a superposition of three Dirac measures. In particular, there is the following relation between the functions $u$ and $v$ :

$$
u=\lambda_{1} \beta_{1}(v)+\lambda_{0} \beta_{0}(v)+\lambda_{2} \beta_{2}(v),
$$




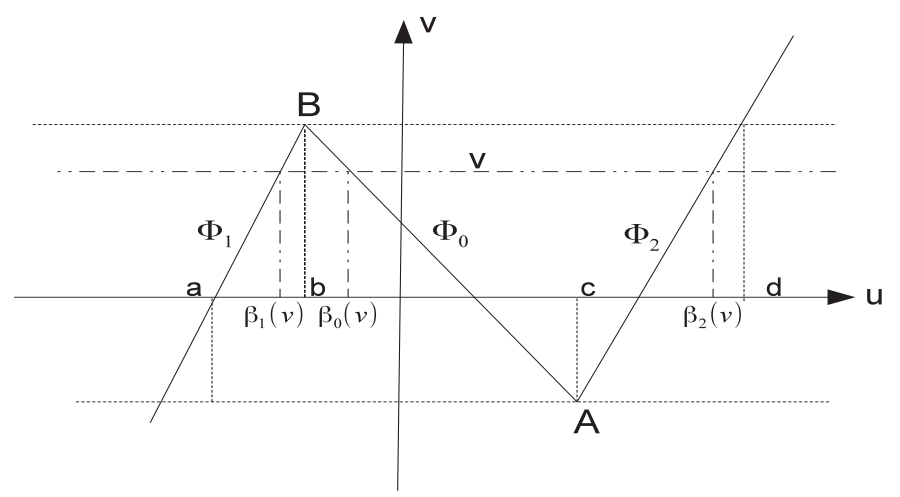

FIG. 1. The function $\phi$

where $\lambda_{i}(x, t) \geqslant 0, i=0,1,2, \sum_{i=0}^{2} \lambda_{i}(x, t)=1$ in $Q_{T}, \beta_{i}(s)$ correspond to the three monotone branches of the graph $s=\phi(u)$ (see Fig. 1). From a physical point of view this fact could be interpreted as a superposition of different phases where $\lambda_{i}$ represents the fraction of phase $i$.

Therefore it can be proved that the equation $u_{t}=\phi(u)_{x x}$ is satisfied in the sense of the measure-valued solutions (see [28], [35]).

We can guess that the complex structure of the solution is due to the presence of the unstable phase. Hence, if we suppose that the initial datum takes values only in the two stable phases, we can assume that $\lambda_{0} \equiv 0$ and the fractions $\lambda_{1}, \lambda_{2}$ are suitable characteristic functions. Obviously in this situation $v=\phi(u)$.

In light of these considerations the "two-phase problem" was introduced. In this setting we suppose that initial datum $u_{0} \in L^{\infty}(\mathbb{R})$ satisfies

$$
\left\{\begin{array}{l}
u_{0} \leqslant b \text { in }(-\infty, 0), \\
u_{0} \geqslant c \text { in }(0, \infty), \\
\phi\left(u_{0}\right) \in H_{l o c}^{1}(\mathbb{R}) .
\end{array}\right.
$$

Let us note that these assumptions assure that initial condition $u_{0}$ is discontinuous only in the point $x_{0}=0$ and $\phi\left(u_{0}\right)$ is continuous everywhere.

We search a solution that satisfies the entropy formulation of Plotnikov and has a particular structure. More precisely, since the initial datum $u_{0}$ takes values only in the stable phases, we impose that solutions of the problem (1) are again in these phases with a regular interface separating the domain $Q_{T}$ into two different regions in which the different phases are achieved. Finally, we suppose that equation $u_{t}=\phi(u)_{x x}$ is fulfilled in the weak sense (see Definition 2.2 in Section 2). It is important to underline that the entropy formulation, obtained by the pseudoparabolic approximation (3), gives a strong admissibility condition for the evolution of the interface that separates the two stable phases (see Proposition 2.3). Existence and uniqueness results for the twophase problem was obtained in [29], [43], [41]. Therefore, at least for solutions in the two stable phases, the formulation of the forward-backward problem suggested by Plotnikov is well-defined. In particular, it is worth to note that the solutions obtained in [20] do not satisfy the entropy condition introduced in [35]. We also point out that, in general, the limit of the Cahn-Hilliard approximation does not satisfy those conditions (see $[2,3,17]$ ). 
On the contrary, if we consider initial data in the unstable phase, it is possible to exhibit examples of non-uniqueness for solutions that satisfy the entropy formulation of Plotnikov (see [44]). These examples are obtained by considering solutions that satisfy the forward-backward equation in the measure-valued sense, that means that the coefficients $\lambda_{i}, i=0,1,2$ are non-trivial functions.

As we mentioned before, it is possible to give different types of approximation of the forwardbackward parabolic equation (1). In principle these could give different formulations and therefore different admissible solutions for a given boundary value problem. Nevertheless, it is interesting to compare the formulation given by the viscous approximation, that we consider in this paper, to that obtained in recent papers $([3,17,21,26])$ in which semi-discrete schemes are considered. In particular, it looks like $([17,21])$ that for the two-phase problem the solutions obtained by the two types of regularization coincide. In [21] there are heuristic motivations, numerical experiments and also some analytic results that show that the conditions along the interface for the two-phase problem are equivalent (see [17] for the case of steady interface). It would be interesting to analyze the general situation and in particular the case in which initial datum takes values also in the unstable phase. In our knowledge this problem is still open.

In [3] authors analyze the case of data in the unstable phase and using a semi-discrete scheme they exhibited examples of measure-valued solutions of the parabolic equations (1). Authors proved that the solutions depend on the choice of the approximating sequence of the initial datum. Hence, they obtained non-uniqueness results for a large class of initial data.

In this paper we consider the two-phase problem in which one of the phases is the unstable one. Obviously, since we impose that the forward-backward equation is satisfied at least in the weak sense, we can not obtain existence for a generic initial datum. However this study is interesting for different reasons.

The first one is that this provides a class of solutions that satisfy the entropy formulation of the forward-backward problem with unstable phase. Moreover the resulting solutions satisfy the forward-backward equation in the weak sense and not only in the class of measure-valued solutions.

Other examples of explicit entropy solutions of the forward-backward parabolic equation that take values also in the unstable phase are given in [18], where the "Riemann problem" is considered and a solution is obtained by self-similar methods.

The second one is that this study could give information about uniqueness or non-uniqueness for entropy solutions that still satisfy the original forward-backward equation. This is not a trivial question, since it is well-known (see e.g. [13]) that for backward parabolic problems uniqueness results are still true.

This paper is organized in two further sections.

In Section 2 we shall state the precise definition of entropy solution. In particular we shall give the definition of the two-phase problem. Moreover, we shall recall which are the entropy admissibility conditions along the interface of the two-phase stable-stable problem. Finally we shall characterize the admissibility condition also for the two-phase problem in which one of the two phases is the unstable one.

In Section 3 there is the main result of the paper. We shall analyze existence for the two-phase stable-unstable problem. We shall concentrate to the case in which the response function $\phi$ is piecewise linear. The scope of this study is to give examples of solutions of the forward-backward parabolic problem that take values in stable and unstable regions. Using the results of Section 2 we find an easy condition that assures that the solution is also an entropy solution. We shall search 
solutions by fixing an admissible interface $\xi(t)$ such that $\xi(0)=0$, and considering on the right and on the left hand side respectively a forward and a backward problem. More precisely

$$
\begin{cases}u_{t}=v_{x x} & \text { in } Q_{T}=\mathbb{R} \times(0, T) \\ u(x, 0)=u_{0}(x) & \text { in } \mathbb{R}^{+} \times\{0\} \\ u(x, T)=u_{T}(x) & \text { in }(-\infty, K) \times\{0\},\end{cases}
$$

with

$$
\left\{\begin{array}{l}
u=\beta_{0}(v) \text { in } V_{1}, \\
u=\beta_{2}(v) \text { in } V_{2},
\end{array}\right.
$$

where

$$
\begin{aligned}
& V_{1}:=\left\{(x, t) \in \bar{Q}_{T} \mid-\infty<x<\xi(t), t \in(0, T)\right\}, \\
& V_{2}:=\left\{(x, t) \in \bar{Q}_{T} \mid \xi(t)<x<+\infty, t \in(0, T)\right\} .
\end{aligned}
$$

and $\xi(T)=K$.

Then, we have to glue together solutions of a forward parabolic problem and a backward parabolic problem imposing the Rankine-Hugoniot condition (24) along the given interface. A similar problem was studied in [16] for a Perona-Malik nonlinearity. Authors obtained that the class of initial data for which there exists a regular solution of that forward-backward problem is dense in a strong topology. In order to obtain those results they have to study forward and backward problem separates by a steady interface. Here we have a different kind of response function, moreover we have to treat a moving interface along which the solutions have a jump. Therefore we use completely different technics. In Section 3 we prove that the solution of the problem is strictly related to the solution of the following generalized Abel's equation

$$
\int_{0}^{t} \frac{k_{1}(t, s)}{\sqrt{t-s}} m^{\prime}(s) d s+\int_{t}^{T} \frac{k_{2}(t, s)}{\sqrt{s-t}} m^{\prime}(s) d s=h(t)
$$

where $k_{1}$ and $k_{2}$, are Holder continuous functions of order $\frac{1}{2}, h(t)$ is a function in $C^{\frac{1}{2}}([0, T])$ that depends on the initial data and $m$ is the unknown that determines the values of the solutions along the interface.

This kind of equation was studied in literature (see for instance [27, 38]) and in particular we deduce an "algebraic Noether nature" of equation (58) this is the content of Theorem 3.17.

This allows to conclude that for a large class of initial data we have an entropy solution of two phase stable-unstable problem.

\section{Entropy formulation}

Let us recall the precise definition of entropy solution introduced by Plotnikov (see [28, 34, 35]). Here we consider the 1-dimensional case and the domain $\Omega=\mathbb{R}$.

DeFinition 2.1 An entropy solution to problem (1) in $Q_{T}$ is given by $u, \lambda_{0}, \lambda_{1}, \lambda_{2} \in L^{\infty}\left(Q_{T}\right)$, $v \in L^{\infty}\left(Q_{T}\right) \cap L^{2}\left((0, T), H_{\text {loc }}^{1}(\mathbb{R})\right)$ such that:

(a) $\sum_{i=0}^{2} \lambda_{i}=1, \lambda_{i} \geqslant 0$ and there holds:

$$
u=\sum_{i=0}^{2} \lambda_{i} \beta_{i}(v)
$$


with $\lambda_{1}=1$ if $v<A, \lambda_{2}=1$ if $v>B$;

(b) the couple $(u, v)$ is a weak solution of the equation $u_{t}=v_{x x}$ in $Q_{T}$ :

$$
\iint_{Q_{T}} u \psi_{t}-v \psi_{x} d x d t+\int_{\Omega} u_{0}(x) \psi(x, 0) d x=0
$$

for any $\psi \in C^{1}\left(\bar{Q}_{T}\right), \psi(\cdot, T)=0$ in $\bar{\Omega}$.

(c)

$$
\iint_{Q_{T}}\left\{G^{*} \psi_{t}-g(v) v_{x} \cdot \psi_{x}-g^{\prime}(v)\left|v_{x}\right|^{2} \psi\right\} d x d t \geqslant 0
$$

for any $\psi \in C_{0}^{\infty}\left(Q_{T}\right), \psi \geqslant 0, g \in C^{1}(\mathbb{R}), g^{\prime} \geqslant 0$ where

$$
G^{*}(x, t):=\sum_{i=0}^{2} \lambda_{i} G\left(\beta_{i}(v(x, t))\right) \quad \text { for a.e. }(x, t) \in Q_{T} .
$$

and

$$
G(u):=\int_{k}^{u} g(\phi(s)) d s, k \in \mathbb{R} .
$$

We do not enter in the details of such definition (see, e.g., [28, 35, 44]).

We just mention that the entropy inequality is obtained, formally, observing that the solutions of the viscous regularization satisfy the following inequality

$$
G\left(u_{\epsilon}\right)_{t} \leqslant\left[g\left(v_{\epsilon}\right) v_{\epsilon x}\right]_{x}-g^{\prime}\left(v_{\epsilon}\right)\left|v_{\epsilon_{x}}\right|^{2}
$$

where $v_{\epsilon}=\phi\left(u_{\epsilon}\right)+\epsilon u_{\epsilon t}$. Therefore, the entropy inequality (14) given in Definition 2.1 is consequence of (17) and the characterization of the Young limit associated to any converging viscous sequence (see [35]).

In particular, we point out that the entropy inequality (14) is satisfied by any couple of functions $(u, v)$, obtained by a proper limit of a subsequence of $\left\{\left(u_{\epsilon_{n}}, v_{\epsilon_{n}}\right)\right\}_{n \in \mathbb{N}}$, where $u_{\epsilon_{n}}$ is the solution of the pseudoparabolic problem and $v_{\epsilon_{n}}=\phi\left(u_{\epsilon_{n}}\right)+\epsilon_{n}\left(u_{\epsilon_{n}}\right)_{t}$. This entropy condition implies a monotonicity condition on the coefficients $\lambda_{i}, i=1,2$ (see [28, 35]).

In this paper we are interested in the two-phase problem. Let us introduce the following

DEFINITION 2.2 Let $u_{0} \in L^{\infty}(\mathbb{R})$ be such that

$\left.H_{1}\right) \phi\left(u_{0}\right) \in C(\mathbb{R}), \phi\left(u_{0}\right) \in C^{1}((-\infty, 0)) \cap C^{1}((0, \infty)), \phi\left(u_{0}\right)^{\prime} \in L^{1}((-\infty, 0)) \cap L^{1}((0, \infty)) ;$

$\left.H_{2}\right)$ given the sets $I_{1}=(-\infty, b], I_{0}=[b, c]$ and $I_{2}=[c,+\infty)$

$$
\left\{\begin{array}{l}
u_{0} \in I_{i} \text { in }(-\infty, 0) \\
u_{0} \in I_{j} \text { in }(0, \infty)
\end{array}\right.
$$

where $i, j \in\{0,1,2\}, i \neq j$.

By a two-phase solution to problem (1) we mean a triple $(u, v, \xi)$ such that:

(i) $u \in L^{\infty}\left(Q_{T}\right), v \in L^{2}\left((0, T) ; H_{l o c}^{1}(\mathbb{R})\right), v \in C\left(\bar{V}_{1}\right) \cap C\left(\bar{V}_{2}\right), v_{x} \in L^{\infty}\left((0, T), L^{1}(\mathbb{R})\right)$ and $\xi:[0, T] \rightarrow \bar{\Omega}, \xi \in C^{1}([0, T]), \xi(0)=0, v(\xi(\cdot), \cdot) \in C([0, T])$,

$$
\lim _{\delta \rightarrow 0^{+}} v(\xi(t+\delta), t)=\lim _{\delta \rightarrow 0^{-}} v(\xi(t+\delta), t) \text { for every } t \in(0, T) ;
$$


(ii) we have:

$$
\left\{\begin{array}{l}
u=\beta_{i}(v) \text { in } V_{1}, \\
u=\beta_{j}(v) \text { in } V_{2}
\end{array}\right.
$$

where

$$
\begin{aligned}
& V_{1}:=\left\{(x, t) \in \bar{Q}_{T} \mid-\infty<x<\xi(t), t \in(0, T)\right\}, \\
& V_{2}:=\left\{(x, t) \in \bar{Q}_{T} \mid \xi(t)<x<+\infty, t \in(0, T)\right\},
\end{aligned}
$$

and

$$
\gamma:=\partial V_{1} \cap \partial V_{2}=\{(\xi(t), t) \mid t \in[0, T]\} ;
$$

(iii) $u$ satisfies condition (b) of Definition 2.1;

(iv) $u$ satisfies condition (c) of Definition 2.1.

By construction, a solution of the two-phase problem is also an entropy solution in the sense of Definition 2.1 that has a particular structure. Observe that, the previous definition implies that $\phi(u)=v$ everywhere so the coefficients $\lambda_{k}, k \in\{0,1,2\}$, correspond to characteristic functions. More precisely, $\lambda_{i}=I_{V_{1}}$ and $\lambda_{j}=I_{V_{2}}$, where we denote with $I_{E}$ the characteristic function of the set $E$. In this case $G^{*}$ defined in (15) is equal to $G(u)$.

This kind of problem was studied in different papers. However, only the case in which the initial datum is in the two stable phases $I_{1}$ and $I_{2}$ was evaluated. In [29] and [43] the authors considered the case in which the response function $\phi$ is piecewise linear. Uniqueness and local existence was obtained in [29], global existence was stated in [43]. The general nonlinear case was studied in [41], where uniqueness was established and local existence was obtained for a class of initial data by using directly the approximation problem (3).

In some sense we can think that we have two different parabolic problems in the regions $V_{1}$ and $V_{2}$. These problems are related each other by (13) and (14). The weak equation (13) gives us the equation of the unknown interface $\xi$ and the entropy integral inequality imposes some admissibility conditions on that interface. The piecewise linear case is easier to treat since in the regions $V_{i}$ we have classical heat equations, then we get more regularity for the solutions. In particular, we can assume the existence of the trace of the function $v_{x}$ along the interface. Then, using the weak equation (13), we obtain the following Rankine-Hugoniot equation (see [8, 28])

$$
\xi^{\prime}(t)=\frac{v_{x}(\xi(t)-, t)-v_{x}(\xi(t)+, t)}{u(\xi(t)+, t)-u(\xi(t)-, t)}
$$

where $v_{x}(\xi(t) \pm, t):=\lim _{s \rightarrow \pm 0} v_{x}(\xi(t)+s, t)$ and $u(\xi(t) \pm, t):=\lim _{s \rightarrow \pm 0} u(\xi(t)+s, t)$.

In the nonlinear case, in general, we can not write a Rankine-Hugoniot equation like (23) for the interface. In fact the parabolic equation in the two regions $V_{1}$ and $V_{2}$ could degenerate at values $c$ and $b$. Thus the trace of $v_{x}$ does not exist in the classical sense. This problem can be handled by observing that equation (13) implies that the field $\left(u,-v_{x}\right)$ is divergence-free in the weak sense. Then, using the general theory of divergence-measure vector fields, it is possible to give sense to the trace of the field along an interface. More precisely we obtain the following generalized 
Rankine-Hugoniot equation

$$
\begin{aligned}
\lim _{\delta \rightarrow 0^{+}} \frac{1}{\delta}\left[\int _ { t _ { 1 } } ^ { t _ { 2 } } \int _ { - \delta } ^ { 0 } \left\{\xi^{\prime}(t)\right.\right. & \left.u(y+\xi(t), t)+v_{x}(y+\xi(t), t)\right\} \alpha(t) d y d t \\
& \left.-\int_{t_{1}}^{t_{2}} \int_{0}^{\delta}\left\{\xi^{\prime}(t) u(y+\xi(t), t)+v_{x}(y+\xi(t), t)\right\} \alpha(t) d y d t\right]=0
\end{aligned}
$$

where $\left.\left(t_{1}, t_{2}\right) \subset(0, T)\right)$ and $\alpha$ is any test function in $C_{0}^{1}\left(\left(t_{1}, t_{2}\right)\right)$ (see [41]).

Regarding admissibility conditions for the evolution of the interface $\xi(t)$, we have to consider the entropy inequalities (14). Let us introduce for any $g \in C^{1}(\mathbb{R}), g^{\prime} \geqslant 0$, the vector field $\left(G(u),-[F(v)]_{x}\right)$, where $G(\lambda)$ is defined in (16) and $F(\lambda)$ is any primitive of $g(\lambda)$. Therefore using (14) we obtain that $\left(G(u),-[F(v)]_{x}\right)$ is a measure-valued vector field. Then, again we can write a condition along the interface $\gamma$. More precisely, for any interval $\left(t_{1}, t_{2}\right) \in(0, T)$ there holds (see [41])

$$
\begin{aligned}
\lim _{\delta \rightarrow 0^{+}}\left(\frac { 1 } { \delta } \int _ { t _ { 1 } } ^ { t _ { 2 } } \int _ { - \delta } ^ { 0 } \left\{[F(v)]_{x}(y+\xi(t), t)+\xi^{\prime}(t) G(u(y+\xi(t), t)\} \alpha(t) d y d t\right.\right. \\
\left.\quad-\frac{1}{\delta} \int_{t_{1}}^{t_{2}} \int_{0}^{\delta}\left\{[F(v)]_{x}(y+\xi(t), t)+\xi^{\prime}(t) G(u(y+\xi(t), t))\right\} \alpha(t) d y d t\right) \leqslant 0 .
\end{aligned}
$$

for any test function $\alpha \in C_{c}^{1}\left(\left(t_{1}, t_{2}\right)\right), \alpha \geqslant 0$.

In some sense we can think that (25) is an entropy condition along the interface $\gamma$.

These admissibility conditions are rather strong when the two phases are the stable ones. More precisely we have (see [41] for the proof)

Proposition 2.3 Let us consider the two-phase problem with initial datum $u_{0}$ that satisfies (6). Let $(u, v, \xi)$ be a two-phase entropy solution of problem (1). Then

$$
\xi^{\prime}(t) \begin{cases}\leqslant 0 & \text { if } v(\xi(t), t)=B \\ =0 & \text { if } v(\xi(t), t) \in(A, B) \\ \geqslant 0 & \text { if } v(\xi(t), t)=A\end{cases}
$$

This means that the interface $\xi(t)$ moves only when $\phi(u)=v$ assumes the critical values $A$, $B$ along it. In particular, the interface does not move when $v$ is in $(A, B)$. This allows to prove in $[29,41]$ uniqueness results for the two-phase stable-stable problem.

It is important to note that equation (23) (or its weak version (24)), that gives the evolution of the interface, depends only on the fact that the forward-backward equation is satisfied at least in the weak sense. Therefore this is common in any formulation in which a solution is given by two phases separated by an interface (see for instance [2]). On the contrary, the entropy conditions characterize a solution in a stricter way. In particular, it is easy to observe that the admissibility conditions for the interface given above are completely different to that used in [2], where the authors consider a Cahn-Hilliard approximation of the forward-backward problem.

In the following we shall concentrate to the case in which one of the two phases is the unstable one. This instance was not considered in literature. We shall prove that admissibility condition is weaker in this situation. 
Let us consider an initial datum $u_{0}$ that satisfies

$$
\begin{cases}u_{0} \in(b, c) & \text { in }(-\infty, 0), \\ u_{0} \in[c, \infty) & \text { in }(0, \infty) .\end{cases}
$$

In the next we refer to the two-phase problem with an initial datum of type (27) as the "two-phase stable-unstable problem" and we denote it with TP S-U.

Observe that we have to pay attention to the level set $v=A$. In fact, when $\phi$ is regular, the parabolic equations in the two regions $V_{i}, i=1,2$ have the same behavior of the porous media equation. Then, it could exists a region with positive measure in which $v \equiv A$. In this case the interface $\xi$ is not well defined since it is not clear where is the separation between the phases. For this reason we restrict the choice of the initial data (27) to the case in which $u_{0} \in(b, c)$ for $x \leqslant 0$. When this choice is made, it is possible to fix $T$ small enough, such that $v(\xi(\cdot), \cdot))>A$ in $(0, T)$.

For the TP S-U we have the following admissibility condition

Proposition 2.4 Let us consider an initial data $u_{0}$ that satisfies (27). Then, given a two-phase solution $(u, v, \xi)$ of the TP SU we get

$$
v(\xi(\cdot), \cdot))>A \text { in }\left(t_{1}, t_{2}\right) \subset(0, T) \Longrightarrow \xi^{\prime} \leqslant 0 \text { in }\left(t_{1}, t_{2}\right) \subset(0, T) .
$$

Proof. We use the same techniques introduced in [41]. In order to prove the assertion by contradiction we suppose that there exists an interval $\left(t_{1}, t_{2}\right)$ and $\rho>0$ such that $\xi^{\prime}(t)>0$ and $v(\xi(t), t))>A+2 \rho$ for any $t \in\left(t_{1}, t_{2}\right)$. Then, we can choose $r$ small enough such that $v \geqslant A+\rho$ in the domain

$$
S:=\left\{(x, t) \in Q_{T}: t \in\left(t_{1}, t_{2}\right), \xi(t)-r \leqslant x \leqslant \xi(t)+r\right\} .
$$

Let us choice an increasing function $g$ such that

$$
g= \begin{cases}<0 & \text { in }(-\infty, A+\rho) \\ 0 & \text { in }[A+\rho, \infty)\end{cases}
$$

Moreover we consider

$$
G(s)=\int_{u^{\rho}}^{s} g(\phi(\tau)) d \tau, \quad F(s)=\int_{B}^{s} g(\tau) d \tau,
$$

where $u^{\rho}$ is the point in $(c,+\infty)$ such that $\phi\left(u^{\rho}\right)=A+\rho$.

Then, using the entropy condition (25), we obtain

$$
\begin{aligned}
\int_{t_{1}}^{t_{2}}\left\{G\left(u_{1}(t)\right)-\right. & \left.G\left(u_{2}(t)\right)\right\} \xi^{\prime}(t) \alpha(t) d t \\
& +\lim _{\delta \rightarrow 0^{+}}\left(\frac{1}{\delta} \int_{t_{1}}^{t_{2}}\{F(v(\xi(t), t))-F(v(\xi(t)-\delta, t))\} \alpha(t) d t\right. \\
& \left.-\frac{1}{\delta} \int_{t_{1}}^{t_{2}}\{F(v(\xi(t)+\delta, t))-F(v(\xi(t), t))\} \alpha(t) d t\right) \leqslant 0
\end{aligned}
$$

where $u_{1}(t):=\lim _{s \rightarrow 0^{-}} u(\xi(t)+s, t)$ and $u_{2}(t):=\lim _{s \rightarrow 0^{-}} u(\xi(t)+s, t)$. Observe that by construction we have $F(v(\xi(t) \pm \delta, t))=F(v(\xi(t), t))=G\left(u_{2}(t)\right)=0$ for $\delta \leqslant r$. Moreover, for every 
$t \in\left(t_{1}, t_{2}\right)$, the function $g(\phi(\cdot))$ assumes negative values in a proper subinterval of $\left(u_{1}(t), u^{\rho}\right)$, then we have $G\left(u_{1}(t)\right)=\int_{u^{\rho}}^{u_{1}(t)} g(\phi(s)) d s>0$. Therefore, using $\xi^{\prime}(t)>0$, inequality (28) gives a contradiction.

In the next section we shall focus to the solution of the TP S-U in the case of the piecewise linear function $\phi$ given in (2). As observed in the Introduction, in this context we may assume more regularity for the solution of the two phase problem. More precisely, in the piecewise linear case, we generalize the definition of the two-phase "stable-stable" problem given in [29], [43].

DEFINITION 2.5 Let $\phi$ be the piecewise linear function (2). Let $u_{0}$ be an initial datum that satisfies the hypothesis $\left.H_{1}\right), H_{2}$ ) of Definition 2.2. By a two-phase regular solution of problem (1) we mean a triple $(u, v, \xi)$ that is solution in the sense of Definition 2.2 that satisfy also

(I) $\xi \in C^{\frac{3}{2}}(0, T)$;

(II) $u \in C^{2,1}\left(V_{1}\right) \cap C^{2,1}\left(V_{2}\right), u_{x} \in L^{\infty}\left(Q_{T}\right)$, and for any $t \in(0, T)$ there exist finite the limits

$$
\lim _{\eta \rightarrow 0^{+}} u_{x}(\xi(t) \pm \eta, t)=: u_{x}\left(\xi(t)^{ \pm}, t\right)
$$

This definition is motivated in the piecewise linear case by the existence results obtained in [29] for the two-phase stable-stable problem.

Then, we can prove the following admissibility result for the TP S-U.

Proposition 2.6 Let $\phi$ be the piecewise linear function (2). Let $u_{0}$ satisfies the hypothesis of Definition 2.2 with $u_{0} \in I_{0}$ for $x<0$ and $u_{0} \in I_{2}$ for $x \geqslant 0$. Then for any two-phase regular solution $(u, v, \xi)$ of the TP S-U such that $u \neq \equiv c$ in $Q_{T}$ we have $\xi^{\prime} \leqslant 0$ in $(0, T)$.

Proof. Let us suppose that there exists $\bar{t}$ such that $\xi^{\prime}(\bar{t})>0$. Then if $v(\xi(\bar{t}), \bar{t})>A$ we obtain a contradiction by using Proposition 2.4. Therefore $v(\xi(\bar{t}), \bar{t})=A$. Let us assume that there exists a sequence $\left\{t_{n}\right\}_{n \in \mathbb{N}}$ converging to $\bar{t}$ such that $v\left(\xi\left(t_{n}\right), t_{n}\right)>A$. Again by continuity we have a contradiction. Then the only possibility is that there exists an interval $\left(t_{1}, t_{2}\right)$ containing $\bar{t}$ such that $\xi^{\prime}(\cdot)>0$ and $u(\xi(\cdot), \cdot) \equiv c$ in $\left(t_{1}, t_{2}\right)$. Suppose that there exists $s_{2} \in\left(t_{1}, t_{2}\right)$ such that $u_{x}\left(\xi\left(s_{2}\right)^{+}, s_{2}\right)=0$. Hence, using the well-known results on the maximum principle (see e.g [13]), we get that there is $\bar{s}_{2} \in\left(t_{1}, t_{2}\right)$ such that $u \equiv c$ in $V_{2} \cap \mathbb{R} \times\left[0, \bar{s}_{2}\right]$. Analogously, if there exists $s_{1} \in\left(t_{1}, t_{2}\right)$ such that $u_{x}\left(\xi\left(s_{1}\right)^{+}, s_{1}\right)=0$, thus we can find $\bar{s}_{1} \in\left(t_{1}, t_{2}\right)$ such that $u \equiv c$ in $V_{1} \cap \mathbb{R} \times\left[\bar{s}_{1}, T\right]$. Therefore we deduce that, if $u \neq \equiv c$ in $Q_{T}$, necessarily $u_{x}\left(\xi(t)^{+}, t\right)>0$ or $u_{x}\left(\xi(t)^{-}, t\right)>0$ for every $t \in\left(t_{1}, t_{2}\right)$.

Let us suppose that $u \neq \equiv c$ in $Q_{T}$. This contradicts the Rankine-Hugoniot condition (24). In fact we get

$$
\begin{aligned}
& 0=\int_{t_{1}}^{t_{2}} \alpha(t) \xi^{\prime}(t)(c-c) d t \\
& =\lim _{\delta \rightarrow 0^{+}} \frac{1}{\delta}\left(\int_{t_{1}}^{t_{2}} \alpha(t)((v(\xi(t)-\delta, t)-A)+(v(\xi(t)+\delta, t)-A)) d t\right) \\
& \int_{t_{1}}^{t_{2}} \alpha(t)\left(\left(\gamma_{2} u_{x}\left(\xi(t)^{+}, t\right)-\gamma_{0} u_{x}\left(\xi(t)^{-}, t\right)\right) d t\right.
\end{aligned}
$$

for any nonnegative test function $\alpha(t) \in C_{c}^{1}\left(\left(t_{1}, t_{2}\right)\right)$. 
Since $\gamma_{0}<0$ and $\gamma_{2}>0$ (the coefficients given in (2)) the left hand side in (29) is strictly positive then we obtain a contradiction.

In the following we always assume that $\phi$ is the piecewise function defined in (2).

Using Proposition 2.6, we saw that the admissibility condition for the TP S-U implies that the unstable phase tends to disappear. In the next proposition we shall prove that the condition $\xi^{\prime} \leqslant 0$ is in fact equivalent to the admissibility condition.

Proposition 2.7 Let $u_{0}$ satisfies the hypothesis of Proposition 2.6. Let $(u, v, \xi)$ be a triple that satisfies conditions $i$ ), $i$ i), $i$ i i $)$ of Definition 2.2 and the conditions $I$ ) and $I I$ ) of Definition 2.5, moreover assume that $u \not \equiv c$ in $Q_{T}$. Then the triple $(u, v, \xi)$ is a regular solution of the TP S-U if and only if $\xi^{\prime} \leqslant 0$ in $(0, T)$.

Proof. Using Proposition 2.6 it remains to prove that $\xi^{\prime} \leqslant 0$ in $(0, T)$ implies that the triple $(u, v, \xi)$ satisfies the entropy admissibility condition (14).

First of all, we observe that, the solution is regular in the regions $V_{1}$ and $V_{2}$. Therefore the entropy admissibility condition (14) is equivalent to the admissibility condition (25) along the boundary. Moreover, there exist the traces of the functions $u$ and $v$ along the interface $\gamma$. Let us introduce the notations $u_{1}(t)=u\left(\xi(t)^{-}, t\right), u_{2}(t)=u\left(\xi(t)^{+}, t\right), v_{x}^{1}(t)=v_{x}\left(\xi(t)^{-}, t\right)$, $v_{x}^{2}(t)=v_{x}\left(\xi(t)^{+}, t\right)$. Therefore condition (25) becomes

$$
\begin{aligned}
\int_{0}^{T}\left\{G\left(u_{1}(t)\right)-G\left(u_{2}(t)\right)\right\} & \xi^{\prime}(t) \alpha(t) d t \\
& +\int_{0}^{T}\left(g\left(\phi\left(u_{1}(t)\right)\right) v_{x}^{1}(t)-g\left(\phi\left(u_{2}(t)\right)\right) v_{x}^{2}(t)\right) \alpha(t) d t \leqslant 0 .
\end{aligned}
$$

for any test function $\alpha \in C_{c}^{1}((0, T)), \alpha \geqslant 0$. Let us recall that, for hypothesis, $\phi\left(u_{1}(t)\right)=\phi\left(u_{2}(t)\right)$ for any $t \in(0, T)$.

Then, in order to prove (30) it is enough to check that

$$
h(t):=\left(G\left(u_{1}(t)\right)-G\left(u_{2}(t)\right)\right) \xi^{\prime}(t)+\phi\left(u_{2}(t)\right)\left(v_{x}^{1}(t)-v_{x}^{2}(t)\right) \leqslant 0
$$

for any $t \in(0, T)$.

Let us observe that the Rankine-Hugoniot condition (24) becomes in this "regular" case

$$
\int_{0}^{T} \beta(t) \xi^{\prime}(t)\left(u_{1}(t)-u_{2}(t)\right) d t-\int_{0}^{T} \beta(t)\left(\left(v_{x}^{2}(t)-v_{x}^{1}(t)\right) d t=0\right.
$$

for any test function $\beta \in C_{c}^{1}((0, T))$. Let us consider the set $J:=\left\{t \in(0, T): u_{1}(t) \neq u_{2}(t)\right\}$. We deduce the classical Rankine-Hugoniot condition

$$
\xi^{\prime}(t)=\frac{v_{x}^{2}(t)-v_{x}^{1}(t)}{u_{1}(t)-u_{2}(t)}
$$

for any $t \in J$. 
Therefore, using $\xi^{\prime}(t) \leqslant 0$ and $\phi(s) \leqslant \phi\left(u_{2}(t)\right)=\phi\left(u_{1}(t)\right)$, for any $s \in\left(u_{1}(t), u_{2}(t)\right)$, we get

$$
\begin{aligned}
h(t) & =-\xi^{\prime}(t)\left(G\left(u_{2}(t)\right)-G\left(u_{1}(t)\right)-g\left(\phi\left(u_{2}(t)\right)\right)\left(u_{2}(t)-u_{1}(t)\right)\right) \\
& =-\xi^{\prime}(t)\left(\int_{u_{1}(t)}^{u_{2}(t)} g(\phi(s))-g\left(\phi\left(u_{2}(t)\right)\right) d s\right) \leqslant 0
\end{aligned}
$$

for any $t \in J$. Let us prove that $\bar{J}=[0, T]$. Suppose that this is not true, then there exists an interval $I=\left(t_{1}, t_{2}\right)$ such that $u_{1} \equiv u_{2} \equiv c$ in $I$. Therefore, reasoning as in the proof of Proposition 2.6, we obtain $u \equiv c$ in $Q_{T}$. This contradicts the hypothesis.

\section{Existence results for two-phase stable-unstable problem}

In this section we analyze the existence of solutions of the TP S-U. Obviously we can not expect to have solutions for general initial data. In fact, in the left hand side of the interface $\gamma$, we have to consider a backward parabolic problem.

Here, we are interested in obtaining explicit solutions of the TP S-U without imposing the initial condition in the semiaxis $x \leqslant 0$. This problem is not well-posed since uniqueness could not be true. In any case it is interesting to study the inverse problem in which we impose the final data $u(\cdot, T)$ in the backward region.

Therefore, we search regular solutions of the two phase stable-unstable problem that satisfy all the conditions of Definition 2.5 but we replace initial condition $u(x, 0)=u_{0}(x)$ for $x \leqslant 0$ with $u(x, T)=u_{T}(x)$ for $x \in(-\infty, K]$, where $K \leqslant 0$ is a fixed constant ad $u_{T}$ a fixed datum that takes values in the unstable phase.

In the following we assume more regularity for the interface $\xi$ and data $u_{0}, u_{T}$. More precisely we give the following definition

Definition 3.1 Let $K$ be a fixed constant in $(-\infty, 0]$. Let $u_{0} \in L^{\infty}\left(\mathbb{R}^{+}\right)$such that $u_{0} \in C^{2}\left(\mathbb{R}^{+}\right)$, $u_{0}^{\prime} \in L^{1}\left(\mathbb{R}^{+}\right), u_{0}^{\prime \prime} \in L^{\infty}\left(\mathbb{R}^{+}\right), u_{0} \geqslant c$ in $\mathbb{R}$. Let $u_{T} \in C^{2}((-\infty, K)), u_{T}^{\prime} \in L^{1}(-\infty, K)$, $u_{T}^{\prime \prime} \in L^{\infty}(-\infty K), u_{T} \in(b, c)$ in $(-\infty, K]$. By a regular two phase entropy solution of the problem

$$
\begin{cases}u_{t}=\phi(u)_{x x} & \text { in } Q_{T}=\mathbb{R} \times(0, T) \\ u(x, 0)=u_{0}(x) & \text { in } \mathbb{R}^{+} \times\{0\} \\ u(x, T)=u_{T}(x) & \text { in }(-\infty, K) \times\{0\},\end{cases}
$$

we mean a triple $(u, v, \xi)$ such that:

(i) $\left.u \in L^{\infty}\left(Q_{T}\right), v \in L^{2}\left((0, T) ; H_{l o c}^{1}(\mathbb{R})\right), v \in C\left(\bar{V}_{1}\right) \cap C\left(\bar{V}_{2}\right), v_{x} \in L^{\infty}\left((0, T), L^{1}(\mathbb{R})\right)\right)$ and $\left.\xi \in C^{1+\beta}([0, T]), \beta>\frac{1}{2}, \xi(0)=0, \xi(T)=K, v(\xi(\cdot), \cdot) \in C([0, T])\right)$,

$$
\lim _{\delta \rightarrow 0^{+}} v(\xi(t+\delta), t)=\lim _{\delta \rightarrow 0^{-}} v(\xi(t+\delta), t) \text { for every } t \in(0, T)
$$

(ii) we have:

$$
\left\{\begin{array}{l}
u=\beta_{0}(v) \text { in } V_{1}, \\
u=\beta_{2}(v) \text { in } V_{2}
\end{array}\right.
$$


where

$$
\begin{aligned}
& V_{1}:=\left\{(x, t) \in \bar{Q}_{T} \mid-\infty<x<\xi(t), t \in(0, T)\right\}, \\
& V_{2}:=\left\{(x, t) \in \bar{Q}_{T} \mid \xi(t)<x<+\infty, t \in(0, T)\right\},
\end{aligned}
$$

and

$$
\gamma:=\partial V_{1} \cap \partial V_{2}=\{(\xi(t), t) \mid t \in[0, T]\} ;
$$

$u \in C^{2,1}\left(V_{1}\right) \cap C^{2,1}\left(V_{2}\right), u_{x} \in L^{\infty}\left(Q_{T}\right)$, and for any $t \in(0, T)$ there exist finite the limits

$$
\lim _{\eta \rightarrow 0^{+}} u_{x}(\xi(t) \pm \eta, t)=: u_{x}\left(\xi(t)^{ \pm}, t\right) .
$$

(iii) the function $u$ fulfills the equation $u_{t}=\phi(u)_{x x}$ in the regions $V_{1}$ and $V_{2}$; with $u(\cdot, 0)=u_{0}$ in $\mathbb{R}$ and $u(\cdot, T)=u_{T}$ in $(-\infty, K)$;

(iv) the Rankine-Hugoniot condition (29) is satisfied;

(v) $\xi^{\prime}(t) \leqslant 0$ for every $t \in(0, T)$.

REMARK 3.2 Using Proposition 2.7 it follows that a two phase entropy solution of problem (34) it is a two phase entropy solution of problem (1) with initial data

$$
\bar{u}_{0}= \begin{cases}u_{0} & \text { in } \mathbb{R}^{+} \\ u(\cdot, 0) & \text { in } \mathbb{R}^{-} .\end{cases}
$$

REMARK 3.3 If we assume that $v(\xi(\cdot), \cdot) \neq A$ in $(0, T)$, we can replace the integral RankineHugoniot condition with the more classical condition (23).

The introduction of the previous problem could be useful for studying inverse problems. Nevertheless, the unique scope of this paper is to obtain explicit entropy solutions of the two-phase problem when one of the phases is the unstable one.

We shall see, in general, that if a solution of the two-phase problem (34) exists this is not unique. In order to study this problem we fix a decreasing function $\xi \in C^{1+\beta}((0, T)), \beta>\frac{1}{2}$ and we search an entropy two-phase solution of problem (34) with $K=\xi(T)$. Moreover we suppose that

$$
\sup _{x \in(-\infty, K)} u_{T}(x)<c, \quad \inf _{x \in(0, \infty)} u_{0}(x)>c
$$

and we search a solution such that $v(\xi(t), t) \neq A$ for every $t \in(0, T)$.

In order to consider the two phase problem (34) we need some results on the Dirichlet-toNeumann map in Time-dependent Domains proved in [12] (see also [11]). More precisely

THEOREM 3.4 Let $\xi(t) \in C^{1}((0, T)), q_{0} \in C^{1}([0, \infty))$ such that $q_{0}^{\prime} \in L^{1}((0, \infty)), g_{0} \in$ $C^{1}([0, T])$. Let us denote with $q(x, t)$ the solution of the Dirichlet problem

$$
\begin{cases}q_{t}=q_{x x} & \text { in } V_{\xi} \\ q(\xi(t), t)=g_{0}(t) & \text { in }(0, T) \\ q(x, 0)=q_{0}(x) & \text { in } \mathbb{R}\end{cases}
$$

where $V_{\xi}:=\left\{(x, t) \in \bar{Q}_{T} \mid \xi(t)<x<+\infty, t \in(0, T)\right\}$. Then, the function $f(t):=q_{x}(\xi(t), t)$ is characterized as the solution of the following Volterra's equation

$$
\pi f(t)=N(t)+\int_{0}^{t} K(s, t) f(s) d s
$$


where the integral kernel is defined by

$$
K(s, t)=\frac{\sqrt{\pi}}{2} \frac{\xi(t)-\xi(s)}{t-s} \frac{e^{-\frac{(\xi(t)-\xi(s))^{2}}{4(t-s)}}}{(t-s)^{\frac{1}{2}}}, \quad 0<s<t<T
$$

and the function $N(t)$ that depends on the initial-boundary value is given by

$$
N(t)=\sqrt{\pi}\left[\frac{1}{\sqrt{t}} \int_{0}^{\infty} e^{-\frac{(\xi(t)-x)^{2}}{4 t}} q_{0}^{\prime}(x) d x-\int_{0}^{t} \frac{e^{-\frac{(\xi(t)-\xi(s))^{2}}{4(t-s)}}}{(t-s)^{\frac{1}{2}}} g_{0}^{\prime}(s) d s\right] .
$$

We shall obtain a solution of the two phase problem (34) by imposing the Rankine-Hugoniot condition (23) and the continuity of the function $v=\phi(u)$ along the interface. Using the results of Theorem 3.4 we shall see that the solution of the original problem is associated to a generalized Abel's problem.

We proceed as follows. We have to consider the following parabolic problems

$$
\begin{aligned}
& \begin{cases}u_{t}=\gamma_{0} u_{x x} & \text { in } V_{1}, \\
u(\xi(t), t)=g_{0}^{-}(t) & \text { in }(0, T), \\
u(x, T)=u_{T} & \text { in }(-\infty, K),\end{cases} \\
& \begin{cases}u_{t}=\gamma_{2} u_{x x} & \text { in } V_{2}, \\
u(\xi(t), t)=g_{0}^{+}(t) & \text { in }(0, T), \\
u(x, 0)=u_{0} & \text { in } \mathbb{R},\end{cases}
\end{aligned}
$$

where $V_{1}$ and $V_{2}$ are given in (20), $g_{0}^{+}, g_{0}^{-}$are unknown of the problem that are related each other by the continuity condition $\phi(u(\xi(t)-, t))=\phi(u(\xi(t)+, t))$ in $(0, T)$. More precisely, we have

$$
\gamma_{0} g_{0}^{-}(t)+\delta_{0}=\gamma_{2} g_{0}^{+}(t)+\delta_{2}=: m(t) .
$$

Finally we determinate the unknown function $m(t)$ by using the Dirichlet-Neumann maps associated to the problems (41) and (42) and imposing the Rankine-Hugoniot condition (23). We shall obtain such Dirichlet-Neumann maps by standard change of variable. More precisely, we have

Proposition 3.5 Let $u^{+}$be the solution of the parabolic problem (42). Then the function $f^{+}(t)=$ $u_{x}^{+}(\xi(t), t)=f_{2}\left(\gamma_{2} t\right)$, where $f_{2}$ is the solution of the following Volterra problem

$$
\pi f_{2}(\tau)=N_{2}(\tau)+\int_{0}^{\tau} K_{2}(s, \tau) f_{2}(\tau) d s, \quad 0 \leqslant \tau \leqslant \gamma_{2} T
$$

with the kernel

$$
K_{2}(s, \tau)=\frac{\sqrt{\pi}}{2} \frac{\bar{\xi}(\tau)-\bar{\xi}(s)}{\tau-s} \frac{e^{-\frac{(\bar{\xi}(\tau)-\bar{\xi}(s))^{2}}{4(\tau-s)}}}{(\tau-s)^{\frac{1}{2}}}, \quad 0<s<\tau<\gamma_{2} T,
$$

$\bar{\xi}(\tau)=\xi\left(\frac{\tau}{\gamma_{2}}\right)$ and

$$
N_{2}(\tau)=\sqrt{\pi}\left[\frac{1}{\sqrt{\tau}} \int_{0}^{\infty} e^{-\frac{(\bar{\xi}(\tau)-x)^{2}}{4 \tau}} u_{0}^{\prime}(x) d x-\int_{0}^{\tau} \frac{e^{-\frac{(\bar{\xi}(\tau)-\bar{\xi}(s))^{2}}{4(\tau-s)}}}{(\tau-s)^{\frac{1}{2}}} \frac{g_{0}^{+^{\prime}}\left(\frac{s}{\gamma_{2}}\right)}{\gamma_{2}} d s\right]
$$


Proof. This is consequence of Theorem 3.4.

Let us consider the function $w(y, \tau):=u^{+}\left(y, \frac{\tau}{\gamma_{2}}\right)$ then this is solution of the problem

$$
\begin{cases}w_{\tau}=w_{y y} & \text { in } \bar{V}_{2} \\ w(\bar{\xi}(\tau), \tau)=g_{0}^{+}\left(\frac{\tau}{\gamma_{2}}\right) & \text { in }\left(0, \gamma_{2} T\right) \\ w(y, 0)=u_{0}(y) & \text { in } \mathbb{R}\end{cases}
$$

where $\bar{\xi}(\tau)=\xi\left(\frac{\tau}{\gamma_{2}}\right)$ is defined in $\left(0, \gamma_{2} T\right)$ and

$$
\bar{V}_{2}=\left\{(x, \tau) \in \bar{Q}_{\gamma_{2} T} \mid \bar{\xi}(\tau)<x<+\infty, \tau \in\left(0, \gamma_{2} T\right)\right\}
$$

Therefore $f_{2}(\tau)=w_{x}(\bar{\xi}(\tau), \tau)$ is characterized by Theorem 3.4. Obviously $f^{+}(t)=f_{2}\left(\gamma_{2} t\right)$ then we obtain the thesis.

Analogously we get the Dirichlet-Neumann map for the parabolic problem (41). More precisely Proposition 3.6 Let $u^{-}$be the solution of the parabolic problem (41). Then the function $f^{-}(t)=$ $u_{x}^{-}(\xi(t), t)=-f_{1}\left(\left|\gamma_{0}\right|(T-t)\right)$, where $f_{1}$ is the solution of the following Volterra problem

$$
\pi f_{1}(\tau)=N_{1}(\tau)+\int_{0}^{\tau} K_{1}(s, \tau) f_{1}(\tau) d s, \quad 0 \leqslant \tau \leqslant\left|\gamma_{0}\right| T
$$

with the kernel

$$
K_{1}(s, \tau)=\frac{\sqrt{\pi}}{2} \frac{\underline{\xi}(\tau)-\underline{\xi}(s)}{\tau-s} \frac{e^{-\frac{(\xi(\tau)-\underline{\xi}(s))^{2}}{4(\tau-s)}}}{(\tau-s)^{\frac{1}{2}}}, \quad 0<s<\tau<\left|\gamma_{0}\right| T
$$

$\underline{\xi}(\tau)=K-\xi\left(T-\frac{\tau}{\left|\gamma_{0}\right|}\right)$ and

$$
N_{1}(\tau)=\sqrt{\pi}\left[-\frac{1}{\sqrt{\tau}} \int_{0}^{\infty} e^{-\frac{(\xi(\tau)-x)^{2}}{4 \tau}} u_{T}^{\prime}(K-x) d x+\int_{0}^{\tau} \frac{e^{-\frac{(\xi(\tau)-\xi(s))^{2}}{4(\tau-s)}} g_{0}^{-\prime}\left(T-\frac{s}{\left|\gamma_{0}\right|}\right)}{(\tau-s)^{\frac{1}{2}}} d s\right]
$$

Proof. In this case we consider the function $\theta(y, \tau):=u^{-}\left(K-y, T-\frac{\tau}{\left|\gamma_{0}\right|}\right)$. It is easy to check that $\theta$ satisfies the following parabolic problem

$$
\begin{cases}\theta_{\tau}=\theta_{y y} & \text { in } \bar{V}_{1}, \\ \theta(\underline{\xi}(\tau), \tau)=g_{0}^{-}\left(T-\frac{\tau}{\left|\gamma_{0}\right|}\right) & \text { in }\left(0,\left|\gamma_{0}\right| T\right), \\ \theta(\bar{y}, 0)=u_{0}(K-y) & \text { in } \mathbb{R}\end{cases}
$$

where

$$
\bar{V}_{1}=\left\{(x, \tau) \in \bar{Q}_{\left|\gamma_{0}\right| T} \mid \underline{\xi}(\tau)<x<+\infty, \tau \in\left(0,\left|\gamma_{0}\right| T\right)\right\} .
$$

Again we use Theorem 3.4 and the relation $f^{-}(t)=-f_{1}\left(\left|\gamma_{0}\right|(T-t)\right)$ with $f_{1}(\tau)=\theta_{x}(\underline{\xi}(\tau), \tau)$ to obtain the thesis. 
REMARK 3.7 In order to determinate the unknown $m(t)=v(\xi(t), t))$ we impose the RankineHugoniot condition (23) that for Propositions 3.5, 3.6 becomes

$$
\xi^{\prime}(t)=\frac{\left|\gamma_{0}\right| f_{1}\left(\left|\gamma_{0}\right|(T-t)\right)-\gamma_{2} f_{2}\left(\gamma_{2} t\right)}{g^{+}(t)-g^{-}(t)} .
$$

We shall prove that equation (47) could be written as a generalized Abel's equation.

First of all we have to enunciate this result on the Volterra equation (for a proof see, e.g., [43]). Proposition 3.8 Let $K$ be a continuous function defined in $C_{T}:=\{(t, s) \in(0, T) \times(0, T)$ : $0<s<t\}$ and $M$ be a positive constant such that

$$
|K(t, s)| \leqslant \frac{M}{\sqrt{t-s}} \quad \text { for every }(t, s) \in C_{T} .
$$

Let us consider the operator $L: C([0, T]) \rightarrow C([0, T])$ defined by

$$
L(x(t))=x(t)-\int_{0}^{t} K(t, s) x(s) d s .
$$

Then, there exists a continuous function $\mathcal{H}(t, s)$ defined in $C_{T}$ such that

$$
x(t)=L(x(t))+\int_{0}^{t} \mathcal{H}(t, s) L(x(s)) d s,
$$

with $\mathcal{H}(t, s)=K(t, s)+G(t, s)$ in $C_{T}$ and $G$ continuous in $\bar{C}_{T}$.

REMARK 3.9 Following the proof given in [43] (Lemma 2.7) we obtain that

$$
G(t, s)=\sum_{i=2}^{+\infty} H_{i}(t, s)
$$

where

$$
H_{1}(t, s)=K(t, s) I_{(0, t)}(s),
$$

(we denote, again, with $I_{E}$ the characteristic function of the set $E$ ) and $H_{n}, n \geqslant 2$ are defined recursively by

$$
H_{n}(t, s)=\int_{0}^{T} H_{n-1}(t, z) H_{1}(z, s) d z .
$$

Moreover the convergence in (50) is uniform in $[0, T] \times[0, T]$.

More precisely in [43] it is proved by induction that

$$
\left|H_{n+2}(t, s)\right| \leqslant \frac{M^{n+2} \pi^{\frac{n+1}{2}} \Gamma\left(\frac{1}{2}\right)}{\Gamma\left(\frac{2+n}{2}\right)}(t-s)^{\frac{n}{2}} I_{(0, t)}(s)
$$

for every $n \in \mathbb{N}$, where $\Gamma$ is the classical Gamma function.

Let us put $h_{1}(t, s)=H_{1}(t, s) \sqrt{t-s}$, suppose that there exist $\alpha<\frac{1}{2}$ and $S>0$ such that

$$
\left|\partial_{t} h_{1}(t, s)\right|,\left|\partial_{s} h_{1}(t, s)\right| \leqslant \frac{S}{(t-s)^{\alpha}} \text { in } C_{T}
$$


then using the same technics that give the estimates (52) we obtain that there exists a constant $\bar{S}$ such that

$$
\left|\partial_{t} G(t, s)\right|,\left|\partial_{s} G(t, s)\right| \leqslant \frac{\bar{S}}{(t-s)^{\frac{1}{2}}} \text { in } C_{T} .
$$

Using Proposition 3.8 we can give the functions $f_{1}$ and $f_{2}$ in the Volterra equation (43) and (45) in a more explicit way. More precisely we have

Proposition 3.10 There exists an Holder continuous function $G_{2}(t, s)$, of order $\frac{1}{2}$, defined in $\bar{C}_{\gamma_{2} T}$, satisfying (54), such that

$$
f_{2}(t)=\frac{N_{2}(t)}{\pi}+\frac{1}{\pi^{2}} \int_{0}^{t}\left(K_{2}(t, s)+G_{2}(t, s)\right) N_{2}(s) d s \text { in }\left(0, \gamma_{2} T\right)
$$

where $f_{2}$ and $N_{2}$ are the functions defined in Proposition 3.6.

Proof. It is enough to use Proposition 3.8 and Remark 3.9. In fact by a straightforward calculation we obtain that there exists a constant $M_{2}$ such that

$$
K_{2}(s, t) \leqslant \frac{M_{2}}{\sqrt{t-s}} \text { in } C_{\left|\gamma_{2}\right| T}
$$

Moreover, since $\xi \in C^{1+\beta}, \beta>\frac{1}{2}$ we obtain that $h_{1}(t, s)=\sqrt{t-s} K_{2}(t, s)$ satisfies estimate (53) with $\alpha=1-\beta<\frac{1}{2}$. Therefore the thesis is consequence of the equation (43)

Analogously we get

Proposition 3.11 There exists an Holder continuous function $G_{1}(t, s)$, of order $\frac{1}{2}$, defined in $\bar{C}_{\left|\gamma_{0}\right| T}$, satisfying (54), such that

$$
f_{1}(t)=\frac{N_{1}(t)}{\pi}+\frac{1}{\pi^{2}} \int_{0}^{t}\left(K_{1}(t, s)+G_{1}(t, s)\right) N_{1}(s) d s \text { in }\left(0,\left|\gamma_{0}\right| T\right)
$$

where $f_{1}$ and $N_{1}$ are the functions defined in Proposition 3.5.

Using the previous results the Rankine-Hugoniot equation (47) becomes

$$
\begin{aligned}
\xi^{\prime}(t) & \left(\frac{m(t)-\delta_{2}}{\gamma_{2}}-\frac{m(t)-\delta_{0}}{\gamma_{0}}\right) \\
=\frac{\left|\gamma_{0}\right| N_{1}\left(\left|\gamma_{0}\right|(T-t)\right)}{\pi}+ & \frac{\left|\gamma_{0}\right|}{\pi^{2}} \int_{0}^{\left|\gamma_{0}\right|(T-t)}\left(K_{1}\left(\left|\gamma_{0}\right|(T-t), s\right)+G_{1}\left(\left|\gamma_{0}\right|(T-t), s\right)\right) N_{1}(s) d s \\
& \quad-\frac{\gamma_{2} N_{2}\left(\gamma_{2} t\right)}{\pi}-\frac{\gamma_{2}}{\pi^{2}} \int_{0}^{\gamma_{2} t}\left(K_{2}\left(\gamma_{2} t, s\right)+G_{2}\left(\gamma_{2} t, s\right)\right) N_{2}(s) d s
\end{aligned}
$$

for every $t \in(0, T)$. Let us observe that $N_{1}$ and $N_{2}$ depend on the unknown $m(t)$. Then we have to analyze in detail the previous equation in order to state that this is equivalent to an Abel's type equation for the function $m^{\prime}(t)$. This is the content of the following 
THEOREM 3.12 Equation (57) is equivalent to the following generalized first order Abel's equation:

$$
\int_{0}^{t} \frac{k_{1}(t, s)}{\sqrt{t-s}} m^{\prime}(s) d s+\int_{t}^{T} \frac{k_{2}(t, s)}{\sqrt{s-t}} m^{\prime}(s) d s=h(t)
$$

where $k_{1}$, respectively $k_{2}$, is an Holder continuos function of order $\frac{1}{2}$ defined in the set $\bar{C}_{T}$, respectively $[0, T] \times[0, T] \backslash C_{T}$ and $h(t)$ is a function in $C^{\frac{1}{2}}([0, T])$ that depends on the initial data $u_{0}^{\prime}, u_{T}^{\prime}$ and $m(0)$.

In order to prove the previous theorem we introduce the following lemmas.

LEMMA 3.13 Let $g_{1}, g_{2}$ Holder continuous functions of order $\gamma$ defined in $C_{T}$. Let us consider the function

$$
h(t, s)=\int_{s}^{t} \frac{g_{1}(t, z) g_{2}(z, s)}{\sqrt{t-z} \sqrt{z-s}} d z,
$$

then $h$ is Holder continuous of order $\gamma$ in $C_{T}$.

Let us fix $(t, s) \in C_{T}$, consider for every $\tau \in(s-t, T-t)$ the following change of variable $w=f(w)=s+(w-s) \frac{t-s+\tau}{t-s}$. We get

$$
h(t+\tau, s)=\int_{s}^{t} \frac{g_{1}(t+\tau, f(w)) g_{2}(f(w), s)}{\sqrt{t-w} \sqrt{w-s}} d w
$$

Therefore

$$
h(t+\tau, s)-h(t, s)=\int_{s}^{t} \frac{g_{1}(t+\tau, f(w)) g_{2}(f(w), s)-g_{1}(t, w) g_{2}(w, s)}{\sqrt{t-w} \sqrt{w-s}} d w .
$$

Let us estimate the numerator in the integrand of (59). We get

$$
\begin{aligned}
\mid g_{1}(t & +\tau, f(w)) g_{2}(f(w), s)-g_{1}(t, w) g_{2}(w, s) \mid \\
& \leqslant\left|g_{2}(f(w), s)\right|\left|g_{1}(t+\tau, f(w))-g_{1}(t, w)\right|+\left|g_{1}(t, w)\right|\left|g_{2}(f(w), s)-g_{2}(w, s)\right| \\
& \leqslant\left|g_{2}(f(w), s)\right|\left(\left|g_{1}(t+\tau, f(w))-g_{1}(t, f(w))\right|+\left|g_{1}(t, f(w))-g_{1}(t, w)\right|\right) \\
& +\left|g_{1}(t, w)\right|\left|g_{2}(f(w), s)-g_{2}(w, s)\right| .
\end{aligned}
$$

Since $g_{1}$ and $g_{2}$ are Holder continuous, we obtain

$$
\begin{aligned}
\left|g_{1}(t+\tau, f(w)) g_{2}(f(w), s)-g_{1}(t, w) g_{2}(w, s)\right| & \leqslant P\left(|\tau|^{\gamma}+|f(w)-w|^{\gamma}\right) \\
& =P\left(|\tau|^{\gamma}+\left|\frac{\tau(w-s)}{t-s}\right|^{\gamma}\right) \\
& \leqslant 2 P|\tau|^{\gamma}
\end{aligned}
$$

where $P$ is a constant that does not depend on $s, t, \tau$. This implies that $h(\cdot, s)$ is Holder continuous of order $\gamma$ uniformly in $s$. Analogously we can prove that $h(t, \cdot)$ is Holder continuous of order $\gamma$ uniformly in $t$. 
Lemma 3.14 Let $\xi \in C^{1+\beta}([0, T]), \beta>\frac{1}{2}, f \in C^{\frac{1}{2}}([0, T])$. Let us consider the following function

$$
g(t):=\int_{0}^{t} K(t, s) f(s) d s,
$$

where

$$
K(t, s)=\frac{\xi(t)-\xi(s)}{t-s} \frac{e^{-\frac{(\xi(t)-\xi(s))^{2}}{4(t-s)}}}{(t-s)^{\frac{1}{2}}} .
$$

Then $g \in C^{\frac{1}{2}}([0, T])$.

Proof. Let $t_{1}, t_{2} \in[0, T]$. It is not restrictive to assume $t_{1}<t_{2}$. By changing variable, we have

$$
g(t)=\int_{0}^{t} \frac{\xi(t)-\xi(t-y)}{y^{\frac{3}{2}}} e^{-\frac{(\xi(t)-\xi(t-y))^{2}}{4 y}} f(t-y) d y .
$$

In order to simplify the notations we introduce the functions

$$
g_{1}(t, y)=\frac{\xi(t)-\xi(t-y)}{y^{\frac{3}{2}}}, g_{2}(t, y)=e^{-\frac{(\xi(t)-\xi(t-y))^{2}}{4 y}}
$$

defined in $C_{T}$. Then

$$
\begin{aligned}
\left|g\left(t_{2}\right)-g\left(t_{1}\right)\right|=\mid \int_{t_{1}}^{t_{2}} g_{1}\left(t_{2}, y\right) g_{2}\left(t_{2}, y\right) f\left(t_{2}-y\right) d y & \\
& \quad+\int_{0}^{t_{1}} g_{1}\left(t_{2}, y\right) g_{2}\left(t_{2}, y\right) f\left(t_{2}-y\right)-g_{1}\left(t_{1}, y\right) g_{2}\left(t_{1}, y\right) f\left(t_{1}-y\right) \mid .
\end{aligned}
$$

Observe that, by hypothesis, there exists a constant $H_{1}$ such that

$$
\left|g_{1}\left(t_{2}, y\right) g_{2}\left(t_{2}, y\right) f\left(t_{2}-y\right)-g_{1}\left(t_{1}, y\right) g_{2}\left(t_{1}, y\right) f\left(t_{1}-y\right)\right| \leqslant \frac{H_{1}}{\sqrt{y}}
$$

then

$$
\left|\int_{t_{1}}^{t_{2}} g_{1}\left(t_{2}, y\right) g_{2}\left(t_{2}, y\right) f\left(t_{2}-y\right) d y\right| \leqslant H_{2} \sqrt{t_{2}-t_{1}},
$$

where $\mathrm{H}_{2}$ is a proper constant.

Let us consider in $\left(0, t_{1}\right)$ the term

$$
\begin{aligned}
g_{1}\left(t_{2}, y\right) g_{2}\left(t_{2}, y\right) f\left(t_{2}-y\right)-g_{1}\left(t_{1}, y\right) & g_{2}\left(t_{1}, y\right) f\left(t_{1}-y\right) \\
=g_{1}\left(t_{1}, y\right) g_{2}( & \left(t_{1}, y\right)\left(f\left(t_{2}-y\right)-f\left(t_{1}-y\right)\right) \\
& +\left(g_{1}\left(t_{2}, y\right) g_{2}\left(t_{2}, y\right)-g_{1}\left(t_{1}, y\right) g_{2}\left(t_{1}, y\right)\right) f\left(t_{2}-y\right) .
\end{aligned}
$$

We can choose $H_{3}$ such that

$$
\left|g_{1}\left(t_{1}, y\right) g_{2}\left(t_{1}, y\right)\left(f\left(t_{2}-y\right)-f\left(t_{1}-y\right)\right)\right| \leqslant \frac{H_{3}}{\sqrt{y}} \sqrt{t_{2}-t_{1}},
$$


then there exists a constant $H_{4}$, that does not depend on $t_{1}$, such that

$$
\left|\int_{0}^{t_{1}} g_{1}\left(t_{1}, y\right) g_{2}\left(t_{1}, y\right)\left(f\left(t_{2}-y\right)-f\left(t_{1}-y\right)\right) d y\right| \leqslant \int_{0}^{t_{1}} \frac{H_{1}}{\sqrt{y}} \sqrt{t_{2}-t_{1}} d y \leqslant H_{4} \sqrt{t_{2}-t_{1}} .
$$

It remains to estimate the term

$$
\begin{aligned}
& \left(g_{1}\left(t_{2}, y\right) g_{2}\left(t_{2}, y\right)-g_{1}\left(t_{1}, y\right) g_{2}\left(t_{1}, y\right)\right) f\left(t_{2}-y\right) \\
& =\left[\left(g_{1}\left(t_{2}, y\right)-g_{1}\left(t_{1}, y\right)\right] g_{2}\left(t_{2}, y\right)\right) f\left(t_{2}-y\right) \\
& +\left[\left(g_{2}\left(t_{2}, y\right)-g_{2}\left(t_{1}, y\right)\right] g_{1}\left(t_{1}, y\right)\right) f\left(t_{2}-y\right) .
\end{aligned}
$$

First of all we consider $g_{1}\left(t_{2}, y\right)-g_{1}\left(t_{1}, y\right)$. Let us apply, for every $y \in\left(0, t_{1}\right)$, the Lagrange theorem to the function $s(\cdot, t)=\frac{\xi(\cdot)-\xi(\cdot-y)}{y^{\frac{3}{2}}}$. Then, for every $y \in\left(0, t_{1}\right)$, there exists $t_{y} \in\left(t_{1}, t_{2}\right)$
such that

$$
\left|g_{1}\left(t_{2}, y\right)-g_{1}\left(t_{1}, y\right)\right|=\left|\frac{\xi^{\prime}\left(t^{y}\right)-\xi^{\prime}\left(t^{y}-y\right)}{y^{\frac{3}{2}}}\right|\left|t_{2}-t_{1}\right| \leqslant \frac{H_{5}}{y^{\frac{3}{2}-\beta}}\left|t_{2}-t_{1}\right|
$$

where again $H_{5}$ is a suitable constant. Since $\beta>\frac{1}{2}$ we deduce that there exists a constant $H_{6}$ such that

$$
\left.\mid \int_{0}^{t_{1}}\left[g_{1}\left(t_{2}, y\right)-g_{1}\left(t_{1}, y\right)\right] g_{2}\left(t_{2}, y\right)\right) f\left(t_{2}-y\right) d y\left|\leqslant H_{6}\right| t_{2}-t_{1} \mid .
$$

Finally we have to consider the term $g_{2}\left(t_{2}, y\right)-g_{2}\left(t_{1}, y\right)$. Again we apply the Lagrange theorem to the function $l(\cdot, y)=e^{\frac{-(\xi(\cdot)-\xi(\cdot-y))^{2}}{4 y}}$, obtaining that for every $y \in\left(0, t_{1}\right)$ there exists $t_{y}^{\prime} \in\left(t_{1}, t_{2}\right)$ such that

$$
\left|g_{2}\left(t_{2}, y\right)-g_{2}\left(t_{1}, y\right)\right|=\left|e^{\frac{-\left(\xi\left(t_{y}^{\prime}\right)-\xi\left(t_{y}^{\prime}-y\right)\right)^{2}}{4 y}} \frac{\left(\xi\left(t_{y}^{\prime}\right)-\xi\left(t_{y}^{\prime}-y\right)\right)\left(\xi^{\prime}\left(t_{y}^{\prime}\right)-\xi^{\prime}\left(t_{y}^{\prime}-y\right)\right)}{2 y}\right|\left|t_{2}-t_{1}\right| .
$$

Hence, we deduce

$$
\int_{0}^{t_{1}}\left[\left(g_{2}\left(t_{2}, y\right)-g_{2}\left(t_{1}, y\right)\right] g_{1}\left(t_{1}, y\right)\right) f\left(t_{2}-y\right) d y \leqslant H_{7}\left|t_{2}-t_{1}\right|
$$

with a proper constant $H_{7}$.

Obviously the constants $H_{1}, \ldots, H_{7}$ depend only on the Holder constants of $\xi^{\prime}, f$ and the Lipschitz constant of $\xi$. Therefore, using (61)-(66), we obtain the claim.

Lemma 3.15 Let $\xi \in C^{1+\beta}([0, T]), \beta>\frac{1}{2}, f \in C^{\frac{1}{2}}([0, T])$. Let $K$ the function defined in (60) and $G(t, s)$ the associated function obtained in Proposition 3.8 and Remark 3.9. Then the function

$$
v(t):=\int_{0}^{t} G(t, s) f(s) d s,
$$

is in the space $C^{\frac{1}{2}}([0, T])$.

Proof. We easily have this result. In fact, $\xi \in C^{1+\beta}, \beta>\frac{1}{2}$ implies that $G(\cdot, s)$ is Holder continuous with exponent $\frac{1}{2}$ uniformly with respect to the variable $s$. 
Proof of Theorem 3.10. In order to prove this result we have to separate the terms that depend on $m^{\prime}$ and the terms that do not depend on it. Thus, it is useful to rewrite $N_{1}=N_{1}^{1}+N_{1}^{2}$ and $N_{2}=N_{2}^{1}+N_{2}^{2}$ where $N_{i}^{1}, i=1,2$ depend only on the initial data $u_{0}^{\prime}$ and $u_{T}^{\prime}$. Since $g_{0}^{-\prime}=\frac{m^{\prime}(t)}{\gamma_{0}}$ and $g_{0}^{+^{\prime}}=\frac{m^{\prime}(t)}{\gamma_{2}}$, we get

$$
\begin{aligned}
& N_{1}^{1}(t)=\frac{-\sqrt{\pi}}{\sqrt{t}} \int_{0}^{\infty} e^{-\frac{(\underline{\xi}(t)-x)^{2}}{4 t}} u_{T}^{\prime}(K-x) d x \text { in }\left(0,\left|\gamma_{0}\right| T\right), \\
& N_{2}^{1}(t)=\frac{\sqrt{\pi}}{\sqrt{t}} \int_{0}^{\infty} e^{-\frac{(\bar{\xi}(t)-x)^{2}}{4 t}} u_{0}^{\prime}(x) d x \text { in }\left(0, \gamma_{2} T\right)
\end{aligned}
$$

and

$$
\begin{aligned}
& N_{1}^{2}(t)=-\pi \int_{0}^{t} \frac{e^{-\frac{(\xi(t)-\underline{\xi}(s))^{2}}{4(t-s)}}}{(t-s)^{\frac{1}{2}}} \frac{m^{\prime}\left(T-\frac{s}{\left|\gamma_{0}\right|}\right)}{\left|\gamma_{0}\right|^{2}} d s \text { in }\left(0,\left|\gamma_{0}\right| T\right), \\
& N_{2}^{2}(t)=-\pi \int_{0}^{t} \frac{e^{-\frac{(\bar{\xi}(t)-\bar{\xi}(s))^{2}}{4(t-s)}}}{(t-s)^{\frac{1}{2}}} \frac{m^{\prime}\left(\frac{s}{\gamma_{2}}\right)}{\gamma_{2}^{2}} d s \text { in }\left(0, \gamma_{2} T\right)
\end{aligned}
$$

where $\bar{\xi}$ and $\xi$ are defined in Proposition 3.6 and 3.5.

Let us introduce the following twelve functions

$$
\begin{aligned}
& s_{1}(t)=\frac{\left|\gamma_{0}\right|}{\pi} N_{1}^{1}\left(\left|\gamma_{0}\right|(T-t)\right), \\
& s_{2}(t)=-\frac{\gamma_{2}}{\pi} N_{2}^{1}\left(\gamma_{2} t\right), \\
& s_{3}(t)=\frac{\left|\gamma_{0}\right|}{\pi^{2}} \int_{0}^{\left|\gamma_{0}\right|(T-t)} K_{1}\left(\left|\gamma_{0}\right|(T-t), s\right) N_{1}^{1}(s) d s, \\
& s_{4}(t)=-\frac{\gamma_{2}}{\pi^{2}} \int_{0}^{\gamma_{2} t} K_{2}\left(\gamma_{2} t, s\right) N_{2}^{1}(s) d s, \\
& s_{5}(t)=\frac{\left|\gamma_{0}\right|}{\pi^{2}} \int_{0}^{\left|\gamma_{0}\right|(T-t)} G_{1}\left(\left|\gamma_{0}\right|(T-t), s\right) N_{1}^{1}(s) d s \\
& s_{6}(t)=-\frac{\gamma_{2}}{\pi^{2}} \int_{0}^{\gamma_{2} t} G_{2}\left(\gamma_{2} t, s\right) N_{2}^{1}(s) d s, \\
& s_{7}(t)=\frac{\left|\gamma_{0}\right|}{\pi} N_{1}^{2}\left(\left|\gamma_{0}\right|(T-t)\right), \\
& s_{8}(t)=-\frac{\gamma_{2}}{\pi} N_{2}^{2}\left(\gamma_{2} t\right), \\
& s_{9}(t)=\frac{\left|\gamma_{0}\right|}{\pi^{2}} \int_{0}^{\left|\gamma_{0}\right|(T-t)} K_{1}\left(\left|\gamma_{0}\right|(T-t), s\right) N_{1}^{2}(s) d s, \\
& s_{10}(t)=-\frac{\gamma_{2}}{\pi^{2}} \int_{0}^{\gamma_{2} t} K_{2}\left(\gamma_{2} t, s\right) N_{2}^{2}(s) d s, \\
& s_{11}(t)=\frac{\left|\gamma_{0}\right|}{\pi^{2}} \int_{0}^{\left|\gamma_{0}\right|(T-t)} G_{1}\left(\left|\gamma_{0}\right|(T-t), s\right) N_{1}^{2}(s) d s,
\end{aligned}
$$




$$
s_{12}(t)=-\frac{\gamma_{2}}{\pi^{2}} \int_{0}^{\gamma_{2} t} G_{2}\left(\gamma_{2} t, s\right) N_{2}^{2}(s) d s
$$

Therefore the right hand side of (57) is equal to $\sum_{i=1}^{12} s_{i}(t)$ in $[0, T]$. Observe that the functions $s_{i}$, $i=1, \ldots, 6$ do not depend on the function $m(t)$.

Let us analyze the terms $s_{1}$ and $s_{2}$. It is clear that these have the same regularity of $N_{1}^{1}(t)$ and $N_{2}^{1}(t)$. By changing variable we can write

$$
N_{2}^{1}(t)=C_{2} \int_{\frac{-\xi\left(\frac{t}{\gamma_{2}}\right)}{2 \sqrt{t}}}^{+\infty} e^{-y^{2}} u_{0}^{\prime}\left(2 \sqrt{t} y+\xi\left(\frac{t}{\gamma_{2}}\right)\right) d y
$$

where $C_{2}$ is a proper constant. Then, we easily obtain, using $u_{0}^{\prime \prime} \in L^{\infty}((0, \infty))$ and $\xi(0)=0$, the following estimate

$$
\frac{d}{d t} N_{2}^{1}(t) \leqslant \frac{\bar{C}_{2}}{\sqrt{t}} \text { in }\left(0, \gamma_{2} T\right]
$$

where $\bar{C}_{2}$ is a suitable constant. This implies that $N_{2}^{1}(t)$ is in the space $C^{\frac{1}{2}}\left(\left[0, \gamma_{2} T\right]\right)$.

Analogously with the change of variable $y=\frac{x+\xi\left(T-\frac{t}{\left|\gamma_{0}\right|}\right)-K}{2 \sqrt{t}}$ we get

$$
N_{2}^{1}(t)=C_{1} \int_{\frac{\xi\left(T-\frac{t}{\left.\mid \gamma_{0}\right)}\right)-K}{2 \sqrt{t}}}^{+\infty} e^{-y^{2}} u_{T}^{\prime}\left(-2 \sqrt{t} y+\xi\left(T-\frac{t}{\left|\gamma_{0}\right|}\right)\right) d y
$$

and using the conditions $\xi(T)=K, u_{T}^{\prime \prime} \in L^{\infty}((-\infty, K))$ we obtain that $N_{1}^{1}$ is in the space $C^{\frac{1}{2}}\left(\left[0,\left|\gamma_{0}\right| T\right]\right)$.

In order to prove that the functions $s_{3}$ and $s_{4}$ are in $C^{\frac{1}{2}}([0, T])$ it is enough to apply Lemma 3.14. The Holder continuity of the functions $s_{5}$ and $s_{6}$ are consequence of Lemma 3.15.

Let us analyze the terms $s_{7}, \ldots s_{12}$ involving the unknown function $m(t)$.

We have

$$
s_{7}(t)=-\left|\gamma_{0}\right| \int_{0}^{\left|\gamma_{0}\right|(T-t)} \frac{e^{\frac{-\left(\underline{\xi}\left(\left|\gamma_{0}\right|(T-t)\right)-\xi(s)\right)^{2}}{\left.4\left|\gamma_{0}\right|(T-t)-s\right]}}}{\left(\left|\gamma_{0}\right|(T-t)-s\right)^{\frac{1}{2}}} \frac{m^{\prime}\left(T-\frac{s}{\left|\gamma_{0}\right|}\right)}{\left|\gamma_{0}\right|^{2}} d s .
$$

Introducing the variable $y=T-\frac{s}{\left|\gamma_{0}\right|}$ and using the relation $\underline{\xi}\left(\left|\gamma_{0}\right|(T-z)\right)=K-\xi(z)$ we obtain the following

$$
s_{7}(t)=-\int_{t}^{T} \frac{e^{\frac{-(\xi(y)-\xi(t))^{2}}{4\left[\left(\gamma_{0} \mid(y-t)\right]\right.}}}{\left|\gamma_{0}\right|^{\frac{1}{2}}(y-t)^{\frac{1}{2}}} m^{\prime}(y) d y .
$$

The term $s_{8}$ is given by a similar singular integral kernel. More precisely we have

$$
s_{8}(t)=\int_{0}^{\gamma_{2} t} \frac{e^{\frac{-\left(\bar{\xi}\left(\gamma_{2} t\right)-\bar{\xi}(s)\right)^{2}}{4\left(\gamma_{2} t-s\right]}}}{\left(\gamma_{2} t-s\right)^{\frac{1}{2}}} \frac{m^{\prime}\left(\frac{s}{\gamma_{2}}\right)}{\gamma_{2}} d s .
$$

Again by changing variable and using the definition of $\bar{\xi}$ this becomes

$$
s_{8}(t)=\int_{0}^{t} \frac{e^{\frac{-(\xi(y)-\xi(t))^{2}}{4\left[\gamma_{2} y\right]}}}{\gamma_{2}^{\frac{1}{2}}(t-y)^{\frac{1}{2}}} m^{\prime}(y) d y
$$


Let us consider term $s_{10}(t)$. We have

$$
s_{10}(t)=\frac{1}{\pi} \int_{0}^{\gamma_{2} t} K_{2}\left(\gamma_{2} t, s\right)\left[\int_{0}^{s} \frac{e^{\frac{-(\bar{\xi}(s)-\bar{\xi}(\tau))^{2}}{4(s-\tau)}}}{(s-\tau)^{\frac{1}{2}}} \frac{m^{\prime}\left(\frac{\tau}{\gamma_{2}}\right)}{\gamma_{2}} d \tau\right] d s .
$$

We use the change of variables $s=\gamma y$ and $\tau=\gamma_{2} z$. We obtain

$$
s_{10}(t)=\frac{\gamma_{2}^{\frac{1}{2}}}{\pi} \int_{0}^{t} K_{2}\left(\gamma_{2} t, \gamma_{2} y\right)\left[\int_{0}^{s} \frac{e^{\frac{-\left(\bar{\xi}\left(\gamma_{2} y\right)-\bar{\xi}\left(\gamma_{2} z\right)\right)^{2}}{4 \gamma_{2}(y-z)}}}{(y-z)^{\frac{1}{2}}} m^{\prime}(z) d z\right] d y
$$

Utilizing the definition of $\bar{\xi}$ and changing the order of integration, $s_{10}$ becomes

$$
\frac{\gamma_{2}^{\frac{1}{2}}}{\pi} \int_{0}^{t}\left[\int_{z}^{t} K_{2}\left(\gamma_{2} t, \gamma_{2} y\right) \frac{e^{\frac{-(\xi(y)-\xi(z))^{2}}{4 \gamma_{2}(y-z)}}}{(y-z)^{\frac{1}{2}}} d y\right] m^{\prime}(z) d z
$$

Denoting with $\tilde{K}_{2}(t, z)$ the function in the square brackets of (69), we observe that this is an Holder continuous function defined in $C_{T}$ by Lemma 3.13 .

With similar techniques we can prove that there exist three Holder continuous functions of order $\frac{1}{2} ; \tilde{K}_{1}, \tilde{G}_{1}$, defined in $[0, T] \times[0, T] \backslash C_{T}$ and $\tilde{G}_{2}$ defined in $\bar{C}_{T}$ such that

$$
\begin{aligned}
s_{9}(t) & =\int_{t}^{T} \tilde{K}_{1}(t, z) m^{\prime}(z) d z, \\
s_{11}(t)=\int_{t}^{T} \tilde{G}_{1}(t, z) m^{\prime}(z) d z & \\
s_{12}(t) & =\int_{0}^{t} \tilde{G}_{2}(t, z) m^{\prime}(z) d z .
\end{aligned}
$$

Considering equation (57), we can write $m(t)=m(0)+\int_{0}^{t} m^{\prime}(z) d z$. Therefore the assertion of the Theorem comes from the Holder regularity of the functions $\xi^{\prime}, s_{1}, \ldots, s_{6}$ and from the formulas (67)-(72).

REMARK 3.16 Observe that from the computations given in the proof of Theorem 3.10 we obtain $k_{1}(t, t)=-\frac{1}{\left|\gamma_{0}\right|^{\frac{1}{2}}}$ and $k_{2}(t, t)=\frac{1}{\gamma_{2}^{\frac{1}{2}}}$. More precisely these values are obtained in (67) and (68).

Generalized Abel's equation like (58) are strictly related to the solutions of singular integral equations. We do not analyze in details this kind of problems we refer to the book of Samko, Kilbas \& Marichev ([38]) for a complete treatise of the subject and to [14] and [27] for the singular integral equations. We just recall that, choosing properly the class of the solutions, it can be proved the Noether nature of the operator

$$
A(\psi)=\int_{0}^{t} \frac{k_{1}(t, s)}{\sqrt{t-s}} \psi(s) d s+\int_{t}^{T} \frac{k_{2}(t, s)}{\sqrt{s-t}} \psi(s) d s .
$$


In particular in [38] it is proved that if $k_{i}$ are Holder continuos of order $\gamma>\frac{1}{2}$, denoting with

$$
I^{\frac{1}{2}}\left(L^{p}(0, T)\right)=\left\{v \in L^{p}(0, T): \text { there exists } \phi \in L^{p}(0, T) \text { such that } v(t)=\int_{0}^{t} \frac{\phi(s)}{\sqrt{t-s}} d s\right\}
$$

then $A: L^{p}(0, T) \rightarrow I^{\frac{1}{2}}\left(L^{p}(0, T)\right)$ is a Noether operator when $p<2$ (see also [37] for a generalization in the case $p \geqslant 2)$.

Unfortunately in our situation $k_{i}$ are Holder continuos at most of order $\frac{1}{2}$ then we can not apply this result. On the other hand, in this case, we can apply a result proved again in [38] which gives the "algebraic Noether nature" of equation (58). In order to enunciate this result we use Remark 3.16 and rewrite equation (58) as

$$
\frac{1}{\sqrt{\gamma_{2}}} \int_{0}^{t} \frac{m^{\prime}(s)}{\sqrt{t-s}} d s-\frac{1}{\sqrt{\left|\gamma_{0}\right|}} \int_{t}^{T} \frac{m^{\prime}(s)}{\sqrt{s-t}} d s+\int_{0}^{T} T(t, s) m^{\prime}(s) d s=h(t)
$$

where $T$ is a regular function in $\{(t, s) \in[0, T] \times[0, T]: t \neq s\}$ which has a singularity along the diagonal of order strictly lesser that $\frac{1}{|t-s|^{\frac{1}{2}}}$. In particular by straightforward calculations it satisfies the hypothesis of Lemma 31.3 in [38].

In this case equation (74) is well posed when $m^{\prime}$ is taken in the space $H^{*}$ and $h$ is in the space $H_{\frac{1}{2}}^{*}$, where

$$
\begin{aligned}
H_{\frac{1}{2}}^{*} & :=\bigcup_{\frac{1}{2}<\lambda \leqslant 1, \epsilon_{1}, \epsilon_{2} \in(0,1)} H_{0}^{\lambda}\left(\epsilon_{1}, \epsilon_{2}\right), \\
H_{0}^{\lambda}\left(\epsilon_{1}, \epsilon_{2}\right) & :=\left\{f(t)=t^{1-\epsilon_{1}}(T-t)^{1-\epsilon_{2}} g(t): g \in C^{\lambda}([0, T]), g(0)=g(T)=0\right\}
\end{aligned}
$$

and

$$
H^{*}:=\bigcup_{0<\lambda \leqslant 1, \epsilon_{1}, \epsilon_{2} \in(0,1)} H_{0}^{\lambda}\left(\epsilon_{1}, \epsilon_{2}\right) .
$$

In some sense the space $H_{\frac{1}{2}}^{*}$ corresponds to the space of Holder continuous functions of order strictly bigger than $\frac{1}{2}$ except on the end points $t=0$ and $t=T$ where the functions can also be singular. Analogously, the space $H^{*}$ corresponds to the space of Holder continuous functions of any arbitrary order except on the end points $t=0$ and $t=T$, in which singularities are allowed. These are good spaces for studying the Abel's equation (74) since it can be proved (see [38]) that the operators

$$
A_{1}(\psi)=\int_{0}^{t} \frac{\psi(s)}{\sqrt{t-s}} d s, A_{2}(\psi)=\int_{t}^{T} \frac{\psi(s)}{\sqrt{s-t}} d s
$$

are well defined from $H^{*}$ to $H_{\frac{1}{2}}^{*}$.

In our case we easily obtain that $h(t)$ is in the space $H_{\frac{1}{2}}^{*}$. In fact, for hypothesis $\xi^{\prime} \in C^{\beta}([0, T])$, with $\beta>\frac{1}{2}$. Moreover we obtained in the proof of Theorem 3.10 that $N_{1}^{1}, N_{1}^{2}$ are not only Holder continuous function of order $\frac{1}{2}$ but also differentiable in $(0,1)$ with derivative that has a singularity of order $\frac{1}{2}$ at the end point $t=0$ or $t=T$. Hence we get that $s_{1}, \cdots s_{6}$ are in the space $H_{\frac{1}{2}}^{*}$.

Therefore we can apply the following result obtained in [38, p. 650, Theorem 31.11] 
THEOREM 3.17 Equation (74) is solvable in $H^{*}$ if and only if

$$
\int_{0}^{T} h(s) \psi_{j}(s) d s=0, j=1, \cdots, k,
$$

where $\psi_{j}$ is a complete system of solutions of the homogenous equation

$$
\frac{1}{\sqrt{\gamma_{2}}} \int_{t}^{T} \frac{m^{\prime}(s)}{\sqrt{t-s}} d s-\frac{1}{\sqrt{\left|\gamma_{0}\right|}} \int_{0}^{t} \frac{m^{\prime}(s)}{\sqrt{s-t}} d s+\int_{0}^{T} T(t, s) m^{\prime}(s) d s=0 .
$$

Here $k$ is the finite dimension of the subspace. Moreover, for every $j=1, \cdots, k, \psi_{j}$ has the following form

$$
\psi_{j}(t)=\frac{\psi_{j}^{*}(t)}{t^{\frac{1}{2}}(T-t)^{\frac{1}{2}}}
$$

with $\psi_{j}^{*}(t)$ an Holder continuous function of any order in $[0, T]$,

The difference between numbers of linearly independent solutions of equations (74) and (75) is equal to 1 .

REMARK 3.18 Observe that, choosing properly the data $u_{0}$ and $u_{T}$, previous result allows to get explicit entropy solutions of the forward-backward parabolic problem in which the presence of the unstable phase is non-trivial. It is important to point out that a solution $(u, v)$ obtained before is also an entropy solution of the original problem (34) only if $v(\xi(t), t) \in(A, B)$ for every $t \in[0, T]$. When such a solution exists Theorem 3.17 gives also non-uniqueness.

In general we can choose $\tau \leqslant T$, such that $v(\xi(t), t) \in(A, B)$ for every $t \in[0, \tau]$. In this manner we have an entropy solution of the forward-backward equation in the strip $\mathbb{R} \times[0, \tau]$.

REMARK 3.19 The case $\xi(\cdot) \equiv 0$ is more easier since we can choose in (74) $T(t, s) \equiv 0$. In this context we can obtain more satisfactory results for the generalized Abel's equation (see [38]).

REMARK 3.20 The analysis handled in this section could be useful to study forward parabolic equation with discontinuous coefficients along a given interface.

\section{REFERENCES}

1. Barenblatt, G. I., Bertsch, M., Dal Passo, R. \& Ughi, M., A degenerate pseudoparabolic regularization of a nonlinear forward-backward heat equation arising in the theory of heat and mass exchange in stably stratified turbulent shear flow. SIAM J. Math. Anal. 24 (1993), 1414-1439. Zb10790. 35054 MR1241152

2. Bellettini, G., Fusco, G. \& Guglielmi, N., A concept of solution and numerical experiments for forward-backward diffusion equations. Discr. Contin. Dyn. Syst. 16 (2006), 783-842. Zb11105. 35007 MR2257160

3. Bellettini, G., Geldhauser, C. \& Novaga, M., Convergence of a semidiscrete scheme for a forward-backward parabolic equation. Adv. Differential Equations 18 (2013), 495-522. Zbl1307. 65118 MR3086463

4. Binder, K., Frisch, H.L. \& JäCKLE, J., Kinetics of phase separation in the presence of slowly relaxing structural variables. J. Chem. Phys. 85 (1986), 1505-1512.

5. Brokate, M. \& Sprekels, J., Hysteresis and Phase Transitions, Applied Mathematical Sciences 121 (Springer, 1996). Zb10951.74002 MR1411908 
6. Bertsch, M., Smarrazzo, F. \& Tesei, A., Pseudoparabolic regularization of forward-backward parabolic equations: A logarithmic nonlinearity. Anal. PDE 3 (2013), 1719-1754. Zbl1284. 35121 MR3148065

7. EwING, R. E., The approximation of certain parabolic equations backward in time by Sobolev equations. SIAM J. Math. Anal. 6 (1975), 283-294. Zbl1115.76048 MR0361447

8. Evans, L.C. \& Portilheiro, M., Irreversibility and hysteresis for a forward-backward diffusion equation. Math. Models Methods Appl. Sci. 14 (2004), 1599-1620. Zbl1064. 35091 MR2103092

9. FIFE, P. C., Models for phase separation and their mathematics. Electron. J. Differential Equations 48 (2000), 26 pp. Zbl0957.35062 MR1772733

10. FRISCH, H. L. \& JÄCKLE, J., Properties of a generalized diffusion equation with memory. J. Chem. Phys. 85 (1986) 1621-1627

11. FoKas, A.S., A Unified Approach to Boundary Value Problems, CBMS-NSF Regional Conference Series in Applied Mathematics, SIAM, (2008). Zb11181. 35002 MR2425886

12. FoKas, A.S. \& Pelloni, B., Generalized Dirichlet-to-Neumann map in time-dependent domains. Stud. Appl. Math. 129 (2012), 51-90. Zb11250.35156 MR2946204

13. Friedman, A., Partial Differential Equations of Parabolic Type (Prentice-Hall, 1991).

14. GaKhov, F. D., Boundary value problems. Translated from the Russian. Reprint of the 1966 translation. Dover Publications, Inc., New York, (1990). Zb10830.30026 MR1106850

15. Ghisi, M. \& Gobbino, M., Gradient estimates for the Perona-Malik equation. Math. Ann. 337 (2007), 557-590. Zbl1130.35081 MR2274543

16. Ghisi, M. \& Gobbino, M., A class of local classical solutions for the one-dimensional Perona-Malik equation. Trans. Amer. Math. Soc. 361 (2009), 6429-6446. Zb11213. 35320 MR2538599

17. Geldhauser, C. \& Novaga, M., A semidiscrete scheme for a one-dimensional Cahn-Hilliard equation.. Interfaces Free Bound. 13 (2011), 327-339. Zbl1238.65092 MR2846013

18. Gilding, B. H. \& TeSEI, A., The Riemann problem for a forward-backward parabolic equation. Phys. D 239 (2010), 291-311. Zbl1190 . 35122 MR2581045

19. Gurtin, M.E., Generalized Ginzburg-Landau and Cahn-Hilliard equations based on a microforce balance. Phys. D 92 (1996), 178-192. Zbl0885. 35121 MR1387065

20. HöLlig, K., Existence of infinitely many solutions for a forward backward heat equation. Trans. Amer. Math. Soc. 278 (1983), 299-316. Zb10524. 35057 MR0697076

21. Helmers, M. \& Herrmann, M., Interface dynamics in discrete forward-backward diffusion equations. Multiscale Model. Simul. 11 (2013), 1261-1297. Zb11302.35199 MR3139607

22. Höllig, K. \& Nohel, J. A., A diffusion equation with a nonmonotone constitutive function. In Systems of nonlinear partial differential equations (Oxford, 1982), pp. 409-422, NATO Adv. Sci. Inst. Ser. C: Math. Phys. Sci., 111 (Reidel, Dordrecht-Boston, Mass., 1983) Zb10531. 35045 MR0725537

23. LAIR, A. V., Uniqueness for a forward backward diffusion equation. Trans. Amer. Math. Soc. 291 (1985), 311-317. Zbl0583.35058 MR0797062

24. LAIR, A. V., Uniqueness for a forward backward diffusion equation with smooth constitutive function. Appl. Anal. 29 (1988), 177-189. Zb10621. 35055 MR0959799

25. Lattes, R. \& Lions, J., Méthode de quasi-réversibilité et applications 15 (1967), Dunod, Paris. Zb10159.20803 MR0232549

26. LafitTe, P. \& MAscia, C., Numerical exploration of a forward-backward diffusion equation. Math. Models Methods. Appl. Sci. 22 (6) (2012) Zb11259.65129 MR2914664

27. Muskhelishvili, N. I., Singular integral equations. Boundary problems of function theory and their application to mathematical physics. Translated from the second (1946) Russian edition. Dover Publications, Inc., New York, (1992) MR1215485

28. Mascia, C., Terracina, A. \& Tesei, A., Evolution of stable phases in forward-backward parabolic 
equations. In Asymptotic Analysis and Singularities (edited by H. Kozono, T. Ogawa, K. Tanaka, Y. Tsutsumi \& E. Yanagida), pp. 451-478, Advanced Studies in Pure Mathematics 47-2 (Math. Soc. Japan, 2007). MR2387251

29. Mascia, C., Terracina, A. \& Tesei, A., Two-phase entropy solutions of a forward-backward parabolic equation . Arch. Rational Mech. 194 (2009), 887-925. Zbl1183. 35163 MR2563628

30. Novick-Cohen, A. \& Pego, R. L., Stable patterns in a viscous diffusion equation. Trans. Amer. Math. Soc. 324 (1991), 331-351. Zb10738. 35035 MR1015926

31. PADRÓN, V., Sobolev regularization of a nonlinear ill-posed parabolic problem as a model for aggregating populations. Comm. Partial Differential Equations 23 (1998), 457-486. Zb10910 35138

32. PADRÓN, V., Effect of aggregation on population recovery modeled by a forward-backward pseudoparabolic equation. Trans. Amer. Math. Soc. 356 (2003), 2739-2756. Zbl1056. 35103 MR2052595

33. Perona, P. \& Malik, J., Scale space and edge detection using anisotropic diffusion. IEEE Trans. Pattern Anal. Mach. Intell. 12 (1990), 629-639.

34. Plotnikov, P. I., Equations with alternating direction of parabolicity and the hysteresis effect. Russian Acad. Sci. Dokl. Math. 47 (1993), 604-608. Zb10831. 35091

35. Plotnikov, P. I., Passing to the limit with respect to viscosity in an equation with variable parabolicity direction. Differential Equations 30 (1994), 614-622. Zb10824. 35100

36. Plotnikov, P. I., Forward-backward parabolic equations and hysteresis. J. Math. Sci. 93 (1999), 747766. Zbl0928.35084 MR1699122

37. RUBIN, B. S., Noether theory for generalized Abel equation with real exponents. Differential Equations 16 (1980), 591-600. Zbl0445. 45006

38. Samko, S. G., Kilbas, A. A. \& Marichev, O. I., Fractional integrals and derivatives. Theory and applications. Translated from the 1987 Russian original. Gordon and Breach Science Publishers, Yverdon, (1993)

39. Slemrod, M., Dynamics of measure valued solutions to a backward-forward heat equation. J. Dynam. Differential Equations 3 (1991), 1-28. Zb10747. 35013 MR1094722

40. Smarrazzo, F., On a class of equations with variable parabolicity direction. Discr. Contin. Dyn. Syst. 22 (2008), 729-758. Zbl1156. 35403 MR2429862

41. Smarrazzo, F. \& Terracina, A., Sobolev approximation for two-phase solutions of forwardbackward parabolic problems. Discrete Contin. Dyn. Syst. 33 (2013), 1657-1697. Zbl1263.35150 MR2995866

42. Smarrazzo, F. \& Tesei, A., Degenerate regularization of forward-backward parabolic equations: The vanishing viscosity limit. Math. Ann. 355 (2013), 551-584. Zbl1264. 35275 MR3010139

43. TERracina, A., Qualitative behavior of the two-phase entropy solution of a forward-backward parabolic problem, SIAM J. Math. Anal. 43 (2011), 228-252. Zbl1244. 35066 MR2765690

44. TERracina, A., Non-uniqueness results for entropy two-phase solutions of forward-backward parabolic problems with unstable phase. J. Math. Anal. Appl. 413 (2014), 963-975. Zb106420307 MR3159815

45. Visintin, A., Forward-backward parabolic equations and hysteresis. Calc. Var. 15 (2002), 115-132. Zbl1010.35056 MR1920717 